Document downloaded from:

http://hdl.handle.net/10251/158179

This paper must be cited as:

Torregrosa, AJ.; Gil, A.; Quintero-Igeño, P.; Tiseira, A. (2019). Enhanced design methodology of a low power stall regulated wind turbine. BEMT and MRF-RANS combination and comparison with existing designs. Journal of Wind Engineering and Industrial Aerodynamics. 190:230-244. https://doi.org/10.1016/j.jweia.2019.04.019

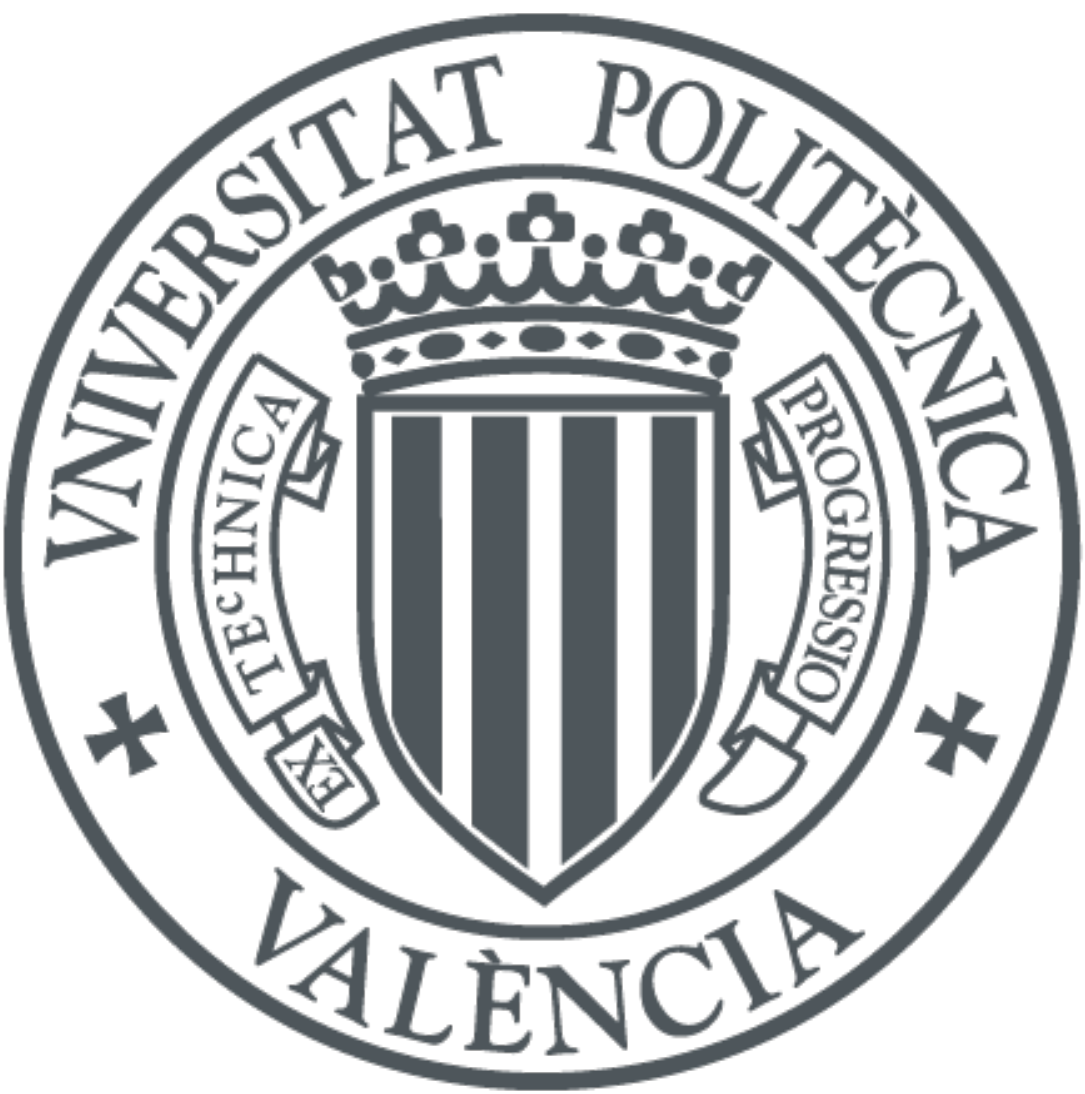

The final publication is available at

https://doi.org/10.1016/j.jweia.2019.04.019

Copyright Elsevier

Additional Information 


\title{
Enhanced design methodology of a low power stall regulated wind turbine. BEMT and MRF-RANS combination and comparison with existing designs
}

\author{
A. J. Torregrosa, A. Gil, P. Quintero*, A. Tiseira
}

CMT-Motores Térmicos, Universitat Politècnica de València, Camino de Vera s/n, 46022 Valencia, Spain.

\begin{abstract}
Wind energy importance has increased over the past decades. Energy generation by small turbines installed near urban locations has experienced noticeable growth. This work is focused on the development of a design methodology for a low power blade well suited for all the wind operation conditions.

First, a complete Design of Experiments will be presented using the low computational cost tool Blade Element Momentum Theory (BEMT) in order to discard those designs which are clearly not suited to the requirements of the system. Later, the remaining were analyzed using a Computational Fluid Dynamics (CFD) methodology in order to account for three dimensional effects. The value of the left slope of the non-dimensional power curve has been found to be a key parameter for the design.

This methodology has been validated with experimental results available from NREL Phase VI wind turbine, allowing to conclude that BEMT is capable to provide with pre-design accurate results which, nevertheless, should corrected by CFD.

The results of the proposed design are analyzed and compared to the CFD predictions of a commercial existing blade designed to comply with similar working. For the proposed design, predictions indicate better behavior in terms of maximum power and controllability.

Keywords: wind turbine, aerodynamics, BEMT, CFD, RANS, fluid dynamics, blade design, design of experiments, MRF, Moving Reference Frame
\end{abstract}

\section{Introduction}

Due to the possible severe climate changes resulting from global warming, there exists a new active interest on the developement of renewable energy. In particular, over the past two decades, the percentage of the energy generated by wind turbines has constantly been increased [1], with an annual average increase of $28 \%$ since the $1990 \mathrm{~s}$. By the 2020s, it is expected that the wind power will generate arround $1200 \mathrm{GW}$, a $12 \%$ of the total energy production. In this context, approximately a $3 \%$ of the total capacity is expected to be introduced in the domestic market [2].

One of the most critical features to be concerned about during the developement of a wind turbine is the blade. After that, the next step is the developement of a control system for the generator and the gear box. Thus, the optimization of both the blade shape and the location of the turbine-farms is of primal interest during the development of new designs [3].

This considerable percentage makes that small wind turbines (SWT) with low maximum power generation (up to $50 \mathrm{~kW}$, in accordance with IEC 61400-2 [4]) are becoming especially interesting. Generally, small wind turbines do not have pitch adjustment to optimize angles of attack at different working conditions being thus necessary to perform a passive optimization for a wide range of wind and rotation velocities.

Due to the characteristics of this kind of wind turbines, which are essentially serial-produced, improvement of the wind turbine blade geometry becomes important even from early stages of the design process. Even though some

*Corresponding author. Tel.: +3496 3877650, fax: +34 963877659.

Email address: pedquiig@mot.upv.es (P. Quintero) 
interesting optimization solutions have been proposed in the literature (see Roshan et al. [5]) their application is rather costly, and thus simpler ways of enhancement are worth to be investigated.

During the design phase, predictions of the wind turbine performance are of interest from cost reduction point of view. In this context, tools requiring low computational cost are normally used. Among these tools, the Blade Element Momentum Theory (BEMT) has been extensively used for studying blade geometries both at design conditions or at off-design (see, for instance Lee et al. [6] or Benini and Toffolo [7]).

The main assumption of BEMT is that the flow can be assumed to be nearly 2D at each airfoil section of the wind turbine. The 2D assumption does not allow to predict the stall-delay phenomenon and could lead to an underestimation of the power generated at near post-stall working points [8]. While some models have been developed to account for this effect [9], here any 3D issues will be considered at a later stage.

BEMT allows to define geometries which maximize the power produced at the design point of the wind turbine [10]. Nevertheless, as the wind turbine needs to behave correctly during some off-design stages, the simple criteria of maximizing the design power is not valid anymore. In this paper, BEMT methodologies are explored and used in order to select a configuration which complies with power and control requirements for a wide range of operating conditions.

When a design is obtained by using BEMT methodologies, the resulting predicted performance needs to be corrected in order to account for possibly important 3D effects [11]. Computational Fluid Dynamics (CFD) is generally used with this aim with different complexity levels. For instance, Tran and Kim [12] performed URANS computations of a complete wind turbine under surge motion and found how the effects of the inclusion of the tower were relatively small in comparison with the mean generated power. Similar conclusions can be extracted from Mo et al. [13], who used Large Eddy Simulation (LES) to study the complete NREL Phase VI wind turbine. Thus, the using of a stationary calculation formulated at a Moving Reference Frame could lead to an important reduction on the computational time.

Note that the previous assumption is only partially valid when dealing with the prediction of the behavior of arrays of wind turbines, as can be deduced from the works of Kang et al. [14] or Santoni et al. [15]. However, it also should be noted that, when dealing with low power wind turbines, they are normally installed in isolated configuration and, furthermore, as their characteristic length is lower, the distance at which wakes are also of important influence will also be lower.

Some studies can also be found in the literature where the elastic deformations of the blades are taken into account. For example, Pourrajabian et al. [16] calculated the flexure solicitations over the blades and they were found to be small compared with the wind turbine dimensions. Similar results can be found in Ponta et al. [17].

About the experimental validation of this kind of flows, the National Renewable Energy Laboratory (NREL) provides with an important amount of information. An important amount of the published literature is based on the validation and comparison of computations with such measured data ([18], [19]). For instance, Esfahanian et al. [20] performed a CFD and BEMT computations over the NREL Phase II wind turbine showing applicability of both methods. Similar conclusions were obtained by Yelmule and EswaraRao [21] for the NREL Phase VI.

This paper presents a proposal of an enhanced blade design methodology which allows definition of designs which are complying not only with the objective of maximizing the produced power under optimum conditions but also introduces additional requirements to consider controllability issues. The design methodology consists on an initial wide discard of possible blade geometries using BEMT, followed by a RANS study, to discard unsuited cases from a reduced number of designs, as will be explained later.

During this work, a classic BEMT methodology was used in order to obtain an enhanced design of a low radius wind turbine. However, BEMT models take into account hypothesis that could lead to inaccurate results, mainly in situations with significant flow separation and when 3D effects become important. As a consequence the final designs must be studied using computational tools which allow to account for these effects, as CFD. Therefore, the main aim of this process is to (a) perform a Design of Experiments (DOE) in order to generate a geometry which is optimum not only from the point of view of maximum possible generation of energy, but also exhibits an acceptable behavior under working conditions out of the design (mainly, blade capability of standing under high winds velocities by means of an improved controllability). For this target BEMT is used for obtaining the features of all the geometries generated during the DOE and to discard those whose behavior is found to be unacceptable. Due to the already commented limitations of this theory, the resulting blade families which were not discarded must be later studied using a three 
dimensional methodology (like RANS) in order to ensure they are still complying with requirements and discard those which do not.

The second aim of the work is (b) compare the resulting design with other commercial wind turbine of similar characteristics both in terms of maximum power generation and controllability.

About the necessity of performing a design that is able to stand under high wind velocity environment, it is known that, for a stall regulated horizontal axis wind turbine (HAWT), it is crucial to have into account the potential risk of circuit burnout or structural failure for wind velocities over a critical value ([21], [22]). In this context, in this article, it will be shown how the left slope of the blade non dimensional power curve is found to be a key design parameter, not fully explored in the literature, in order to obtain a design able to produce power in a wide range of wind velocities avoiding this risk.

In order to assess with this target, the work flow must be structured as sketched by Figure 1. Three main blocks can be identified on the structure of this article: First, it is necessary to develop and validate both a CFD and a BEMT methodology. This will be found during sections 2 and 3, where this validation is performed against the available experimental data about NREL Phase VI.

Once the methodology has been proven to provide with good results, a set of experiments will be designed and calculated by using BEMT, which, due to its affordable computational expense, allows a good first approximation for the behavior of a complete set of candidate geometries. The second step at this stage will be the selection of a reduced family of candidate geometries, which will be analyzed using CFD in order to correct the BEMT results having into account three dimensional effects and discarding those which become unsuited once these computations are performed. This work stage can be found at section 3.1, illustrating only the selection of the final blade and its comparison with CFD. About the use of RANS to discard other blades proposed in this section, reader can refer to the end of the section 4.2 .

Finally, once the final design is proposed, it is necessary to compare it with other existent solutions in order to check its commercial applicability. In this document, the proposed design was compared against one of the leading low power wind turbines, the commercial blade Skystream. As this design is characterized by a curved blade, three dimensional effects will highly affect its behavior, so this will only analyzed via the CFD methodology. The addition of other aerodynamic components, like a Vortex Generator (VG) will be also analyzed and discussed. This stage is developed during the section 4.2 , where reader will also find a detailed discussion of the controllability of the commercial blade, the proposed design and an alternative geometry, which was valid in accordance with BEMT methodology but, when RANS corrections were performed, it was found to not provide with an acceptable behavior in terms of control and, in consequence, was discarded.

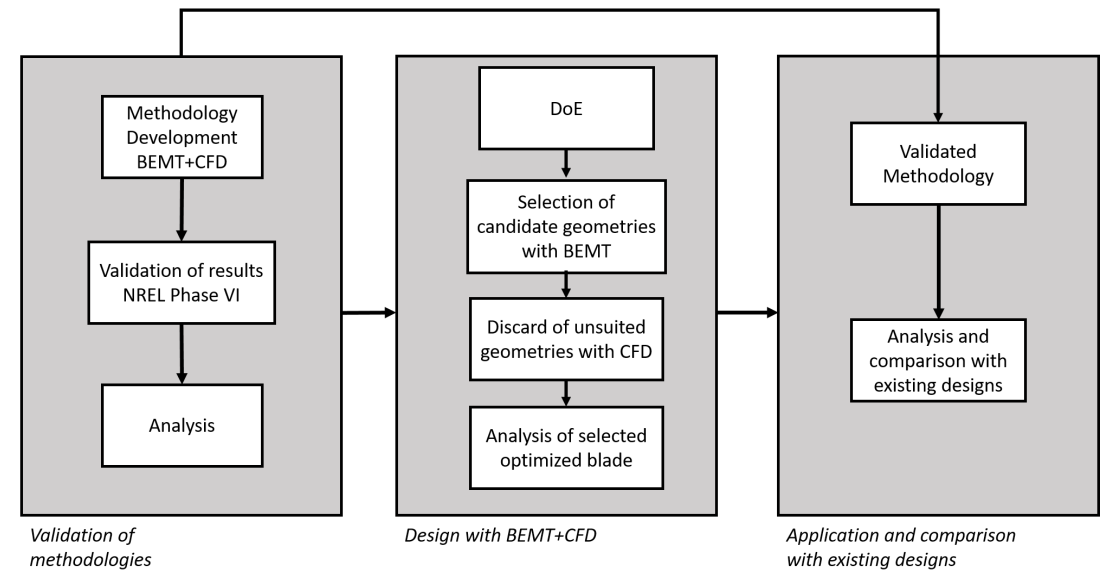

Figure 1: Work flow sketch 


\section{Theoretical Background}

\subsection{Blade Element Momentum Theory}

In this paper, the Blade Element Momentum Theory is used in order to predict the steady-state characteristics of a set of possible blade designs due to its low computational cost. BEMT models have been extensively used and validated in the literature to perform relatively fast/accurate predictions [23].

When the flow is assumed to be nearly 2D, a velocity diagram can be stated for an airfoil of the blade located at a distance $r$ with a pitch angle $\theta$ and a chord $c$ [24], as shown in Figure 2:

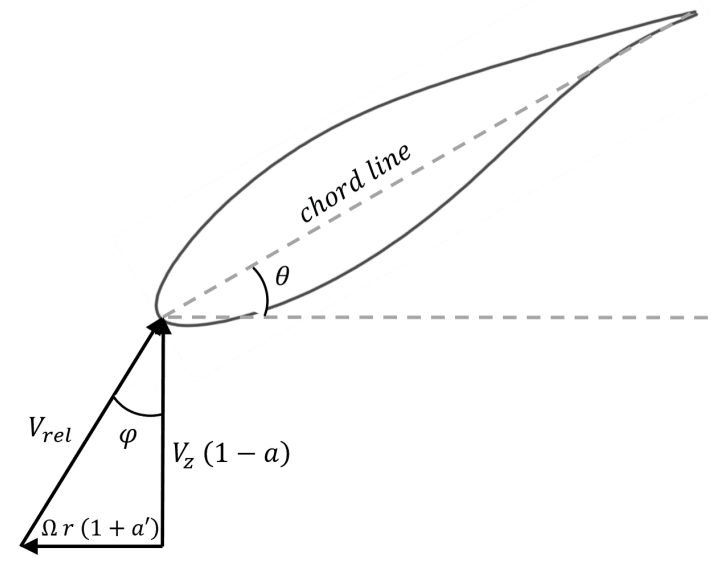

Figure 2: Velocity diagram used for the BEMT explanation at an airfoil section located at radius $r$

Here, $V_{z}$ is the wind velocity and $\Omega$ is the wind turbine rotational velocity, while $a=v_{i} / V_{z}$ and $a^{\prime}=\omega^{\prime} / \Omega$ are the axial and tangential induced velocities, respectively. $V_{\text {rel }}$ is the relative wind velocity magnitude, which shows a relative angle, $\varphi$. These values allow to calculate the angle of attack and the airfoil contribution to the normal force, $F_{N}$, and power, $P$, as follows [10]:

$$
\begin{gathered}
\tan \varphi=\frac{V_{z}}{\Omega r} \frac{1-a}{1+a^{\prime}} \\
\alpha=\varphi-\theta \\
\mathrm{d} F_{N}=\frac{B}{2} \rho V_{r e l}^{2}\left(C_{l} \cos \varphi+C_{d} \sin \varphi\right) c \mathrm{~d} r \\
\mathrm{~d} P=\frac{B \Omega}{2} V_{r e l}^{2}\left(C_{l} \sin \varphi-C_{d} \cos \varphi\right) c r \mathrm{~d} r
\end{gathered}
$$

where $B$ is the number of blades conforming the rotor of the wind turbine and $C_{l}$ and $C_{d}$ are the lift and drag coefficients of the airfoil. These are a function of the angle of attack and can be obtained by means of measurements [25] or computational studies [20].

Once the value of the induced terms $a$ and $a^{\prime}$ is known, the total force and power can be immediately derived as:

$$
F_{N}=\int_{0}^{R} \mathrm{~d} F_{N} \quad ; \quad P=\int_{0}^{R} \mathrm{~d} P
$$

The induced factors can be obtained by means of iterative methods. One of the most popular ones starts with he formulation of the momentum equation for a differential circumference of width $\mathrm{d} r$. Then, introducing the nondimensional tip speed ratio $\lambda=(\Omega R) / V_{z}$ one may write [10]: 


$$
\mathrm{d} C_{T}=\left\{\begin{array}{ll}
8 F a(1-a) x d x & \text { if } a \leq 2 / 5 \\
-(4 / 9) x\left[-4+a(20-18 F)-a^{2}(25-18 F)\right] d x & \text { if } a>2 / 5
\end{array} \quad ; \mathrm{d} C_{P}=8 F x^{3} \lambda^{2} a^{\prime}(1-a)\right.
$$

Here, $C_{T}=2 F_{N} /\left(\rho \pi R^{2} V_{z}^{2}\right), C_{P}=2 P /\left(\rho \pi R^{2} V_{z}^{3}\right)$ and $x=r / R$ are the non-dimensional normal force, power and position, respectively. The coefficient $F$ accounts for the losses at the tip, and one of the most used approximations for its determination is based on Prandlt's method (see de Vries [26]):

$$
F=\frac{2}{\pi} \cos ^{-1}\left[\exp \left(-\frac{B(1-x)}{2 x \sin \varphi}\right)\right]
$$

Once the induction terms are obtained for each section of the blade and equation (2) is integrated, the power and force coefficients of the wind turbine are obtained. An important advantage of the dimensionless form of the equations used is that, for a given pitch and turbine geometry, they are a function only of $\lambda$ and the Reynolds number [27].

\subsection{Reynolds Averaged Navies-Stokes equations}

In this paper, the fluid flow around an optimum blade shape is modeled by means of the Reynolds Averaged Navier-Stokes (RANS) equations formulated on a rotating reference frame. These can be derived from the complete set of mass, momentum and energy conservation equations and are shown next for an incompressible flow [28].

$$
\left\{\begin{array}{c}
\frac{\partial U_{i}}{\partial x_{i}}=0 \\
\frac{\partial U_{i}}{\partial t}+U_{j} \frac{\partial U_{i}}{\partial x_{j}}=v \frac{\partial^{2} U_{i}}{\partial x_{j} \partial x_{i}}-\frac{\partial\left\langle u_{i} u_{j}\right\rangle}{\partial x_{j}}-\frac{1}{\rho} \frac{\partial p}{\partial x_{i}}+f_{i}
\end{array}\right.
$$

where $U_{i}$ represents the component in the $i^{\text {th }}$ direction of the mean velocity field, $\vec{U} ; \rho$ and $v$ are the density and the kinematic viscosity of the fluid, respectively; $p$ represents the average pressure field and $f_{i}$ is the component on the $i^{\text {th }}$ direction of the inertial and body forces acting on the fluid. These terms can be evaluated, when the equations are formulated in a rotating frame, as follows [29]:

$$
\vec{f}=\left(\begin{array}{c}
f_{x} \\
f_{y} \\
f_{z}
\end{array}\right)=-\frac{\mathrm{d} \vec{\omega}}{\mathrm{d} t} \times \vec{r}+\vec{\omega} \times \vec{\omega} \times \vec{r}
$$

where $\vec{\omega}$ represents the rotational velocity of the reference frame, and $\vec{r}$ is the position vector of a point of the fluid.

Closure of equations (5) can only be achieved by modeling the terms $\left\langle u_{i} u_{j}\right\rangle$, which are commonly referred to as the Reynolds stresses. The selection of an appropriate turbulence model is of primal importance for the evaluation of the flow characteristics. In this paper the $k-\omega$ model with shear stress transport (SST) will be used with this purpose. This turbulence model has been extensively used in the literature for this kind of flows, demonstrating an acceptable behavior. As an example, Moshfegui et al. [30] used this model to perform calculations of the NREL Phase VI wind turbine with different near wall grid resolutions, obtaining reasonably good results compared with experimental data [18]-[19]. Similar results were obtained by Yelmule and EswaraRao [21] with the same turbulence model.

The $k-\omega$ SST model was proposed by Menter [31] and is a transitional model in which the formulation considered varies from the $k-\omega$ turbulence model proposed by Wilcox [32] close to the walls and the $k-\varepsilon$ model away from walls, thus solving the main inconveniences of both models. In addition to a transport equation for the turbulent kinetic energy, $k$, the $k-\varepsilon$ and the $k-\omega$ turbulent models solve a transport equation for the turbulent dissipation rate, $\epsilon$, and the specific turbulent dissipation rate, $\omega$, respectively. These variables are related by $\omega \propto \varepsilon / k$ and allow obtaining the turbulent viscosity $v_{t}=C_{\mu} k^{2} / \varepsilon$ (being $C_{\mu}=0.09$ a modeling constant). This turbulent viscosity is used to model the value of the Reynolds stress tensor as:

$$
\left\langle u_{i} u_{j}\right\rangle=\frac{2}{3} \cdot k \cdot \delta_{i j}-v_{T}\left(\frac{\partial\left\langle U_{i}\right\rangle}{\partial x_{j}}+\frac{\partial\left\langle U_{j}\right\rangle}{\partial x_{i}}\right)
$$




\section{Methodology}

\subsection{Design of experiments}

In this section, a geometrical distribution of chord and torsion is proposed and analyzed by using BEMT. An important amount of research has been focused on obtaining an optimum maximum power coefficient for a given working condition. For instance, Burdett and VanTeuren [33] found an optimized solution for a given tip speed ratio assuming that the Reynolds number can affect the 2D airfoil characteristics. It was shown that this influence was practically inappreciable.

For given design values $\alpha_{d}$ and $\lambda_{d}$, Spera [34] demonstrated that optimizing the power coefficient for a blade element is equivalent to find the maximum of the function $f\left(a, a^{\prime}\right)$ defined by:

$$
f\left(a, a^{\prime}\right)=a^{\prime}(1-a)
$$

subject to the condition:

$$
\left(\lambda_{d} x\right)^{2} a^{\prime}\left(1+a^{\prime}\right)=a(1-a)
$$

Once equations (8) and (9) are solved, for a known airfoil section where $C_{l}\left(\alpha_{d}\right) \gg C_{d}\left(\alpha_{d}\right)$, its geometrical parameters can be calculated as follows:

$$
\begin{gathered}
\sigma(x)=\frac{4 a^{\prime}}{1+a^{\prime}} \frac{\sin ^{2} \varphi}{\cos \varphi} \frac{x}{C_{l}\left(\alpha_{d}\right)} \\
\theta(x)=\varphi-\alpha_{d}
\end{gathered}
$$

where $\sigma(x)=B c(x) /(2 \pi R)$ is defined as the local solidity of an airfoil located at an non-dimensional distance $x$ from the hub.

Usually, the value of $\alpha_{d}$ is chosen so as to provide the maximum aerodynamic efficiency, $C_{l} / C_{d}$, at each airfoil section for the design tip speed ratio, $\lambda_{d}$. This approximation also allows to obtain a maximum local value for the power coefficient, but, however, it can lead to unacceptable performance for out-design points.

Due to the cost-performance requirements of low-power wind turbines, it is necessary to minimize the automatic control necessities. Thus, to ensure that the automatic control does not have to apply an unacceptable brake torque at high wind velocities, the turbine must be effectively stall-regulated. As the generated power scales as $P \propto C_{P} V_{z}^{3}$ the necessity of a stall-regulated turbine leads to the condition $\mathrm{d} P / \mathrm{d} V_{z}<0$ for the maximum expected wind velocity. This can be rewritten in terms of only the tip speed ratio and the power coefficient as:

$$
\frac{\mathrm{d} C_{P}}{\mathrm{~d} \lambda}>\frac{3 C_{P}}{\lambda}
$$

Equation (11) indicates that, to ensure controllability at maximum wind speed, the $C_{P}-\lambda$ curve must have a high slope from the left.

To guarantee that both the wind turbine is easily controllable and that its maximum power coefficient is also high, the simple criterion of taking $\alpha_{d}$ for the maximum $C_{l} / C_{d}$ is not sufficiently accurate. Therefore, a design of experiments (DOE) was defined by varying both $\lambda_{d}$ and $\alpha_{d}$ and applying Equation (10). Then, the non-dimensional power curve was obtained for each possible design. An optimum blade was selected ensuring compliance with Equation (11) and considering that the blade should be constructively feasible. The range and step of the parameters considered in the DOE were:

$$
\begin{aligned}
& \lambda_{d} \in[4.5-10.5] \quad ; \quad \Delta \lambda=1 \\
& \alpha_{d} \in[3-12] \mathrm{deg} \quad ; \quad \Delta \alpha=1 \mathrm{deg}
\end{aligned}
$$

The NREL S809 airfoil was selected, as it has been extensively studied and has shown to provide good performance [35]. Also, an important amount of information for a wide range of angles of attack is available [25]. This airfoil will kept for the whole radius of the wind turbine. Using of a single airfoil is a common practice on the design of small wind turbines ([36], [37]). Due to the low size of this kind of wind turbines, all their sections can be considered to work under the range of low Reynolds number and, thus, using different airfoils at root and tips should only marginally increase the performance of the wind turbine. 
Once the DOE methodology has been presented, a complete set of candidate geometries will be generated. A decision process must be then assessed, as sketched by Figure 3. After a selection of a $\lambda_{\text {design }}$ and $\alpha_{\text {design }}$, a possible geometry will be generated, which will be analyzed using BEMT. If the BEMT calculated design complies with certain requirements, which will be explained later, this will be considered as a preliminar candidate and will be analyzed using CFD. If similar restrictions are still complied with, the geometry will be considered as a final candidate. If any of the requirements is not satisfied at any stage, the blade geometry is discarded.

Traditionally, the selection is made mainly by constructive and maximum power criteria. However, for the design of a stall regulated wind turbine it is necessary to have into account that the blade should be easily controllable in accordance with the left slope condition, Equation 11. Note that, with the current methodology, and depending on how the parametric swept is performed, it could be possible that more than one design could arrive to the final candidate phase. If this is the case, all the resulting candidates would be valid design and extra conditions could be imposed to them (for instance, the design with higher maximum power coefficient could be selected). With the conditions of the current work only one geometry complied with the requirements after the CFD decision phase.

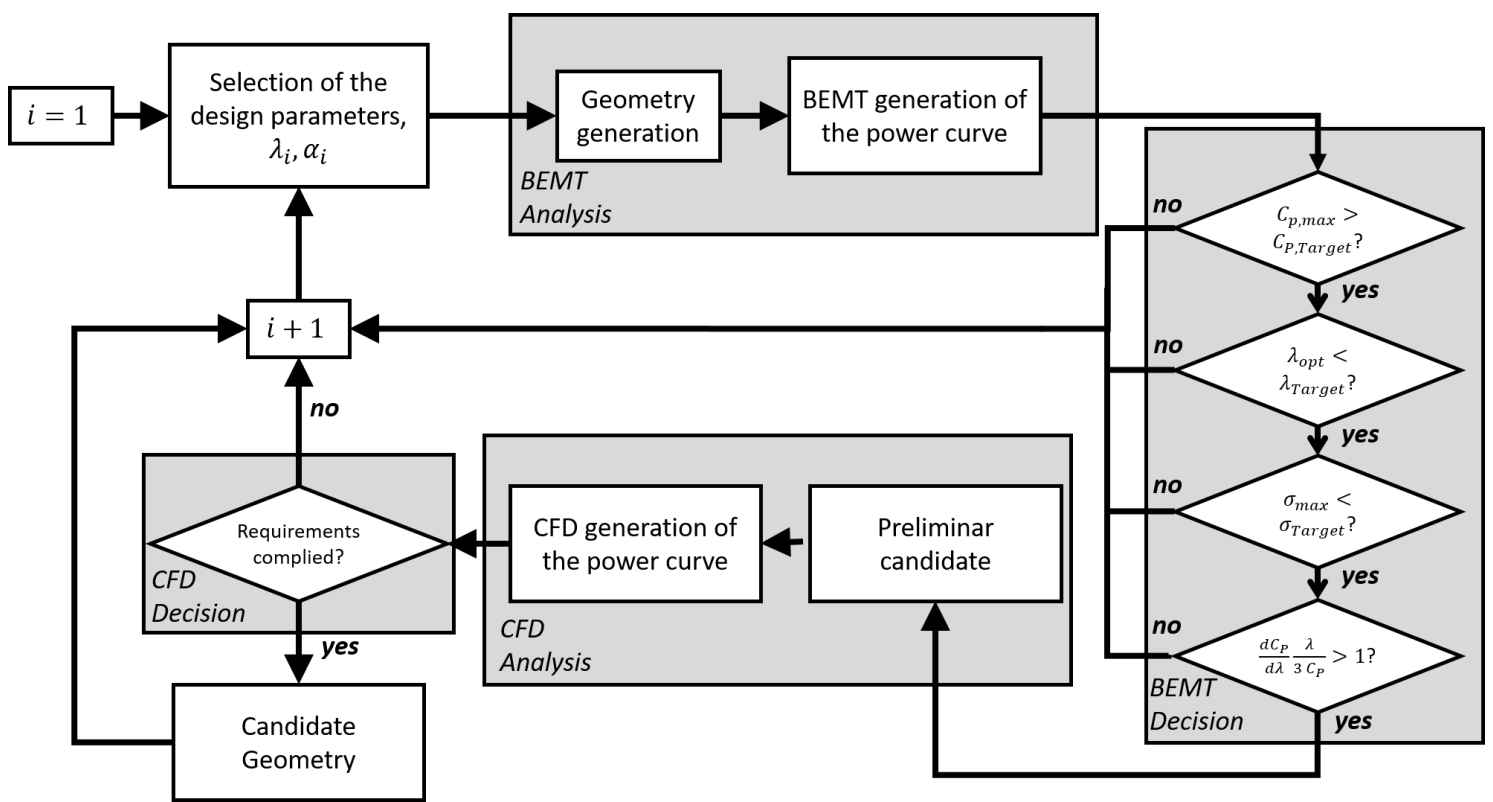

Figure 3: Work flow of the proposed Design of Experiments

Once the Design of Experiments has been qualitatively described, it is necessary to establish which value of the different parameters will be considered. These are summarized next:

- The maximum power coefficient of the new blade design must be high. In this sense it will be consirered that the resulting blade should provide a maximum power coefficient at optimum conditions of $\left(C_{P}\right)_{\text {optim }}>0.37$, which would correspond with a power generation of approximately $P=1500 \mathrm{~W}$ for a blade with radius $R=$ $2.50 \mathrm{~m}$ at a wind velocity of $V_{z}=7 \mathrm{~m} \mathrm{~s}^{-1}$.

- The optimum rotational velocity should be as low as possible in order to minimize radiated noise operating under design conditions. In this sense it will be supposed that an acceptable design should present its maximum power coefficient at $\lambda_{\text {optim }}<6.5$, which would correspond with an optimum rotational velocity of approximately $\Omega=170 \mathrm{rpm}$ at the conditions of the previous point. It will be shown that RANS predictions will be expected to provide a higher estimation of this parameter. In consequence, this constraint will be slightly relaxed during the CFD evaluation as the controllability restrictions will be of higher importance. Note how, even with the mentioned relaxation of this parameter, the operating rotational speed of the RANS predictions will still be kept bounded. 
- As it was previously stated, in order to achieve a geometry which is easily controllable for a stall-regulated blade turbine wind turbine, the left slope of the power curve should be as high as possible. For the purpose of the current design it will be stated that the new wind turbine should comply with $\frac{d C_{P}}{d \lambda} \frac{\lambda}{3 C_{P}}>1$ when $\lambda=0.85 \cdot \lambda_{\text {optim }}$

- The behavior of the wind turbine for very low values of the tip speed ratio should be acceptable in order to ensure a correct starting. Note that this is a qualitative statement. As it is expected that for low values of $\lambda$ the validity of BEMT assumptions are only partially valid, it will also be possible to observe how some BEMT predicted designs exhibit power coefficients which become negative under certain conditions. This obviously will not be the case, but it can be assumed that, even with the taken simplifications, the behavior of this design under low rotational speeds will not be acceptable.

- The manufacturing of the resulting blade turbine should be as simple as possible. In this sense, the maximum local solidity, $\sigma$, (which, as will be seen, will be near to the hub) should never exceed from certain value. If this solidity is too high it will be difficult to construct the transition between it and the hub. Thus, for the current work it will be supposed that the design should comply with $\sigma_{\max }<0.10$.

All the blade geometries complying with the previous constraints will be considered as possible candidates (five candidates, for the constraints already mentioned). These, will be studied via CFD in order to have into account $3 \mathrm{D}$ effects and ensure the so studied design complies with the stated requirements, discarding those which do not. Although this was performed for all the BEMT-suited blades, only the final choice will be shown in section 4.1 , for reasons of brevity. Note also how the second requirement will be slightly relaxed.

\subsection{CFD modeling}

In order to account for the 3D effects of the flow, the set of Equations (5) are numerically solved in a Moving Reference Frame (MRF) by means of the Finite Volume Method, using the general purpose commercial package, STAR CCM+, and using a second order upwind discretization. In order to set up the CFD calculation, the rotor NREL Phase VI was first analyzed and validated against available experimental measurements (see section 3.3). This wind turbine is formed by 2 blades with radius $R=5.11 \mathrm{~m}$. The chord and torsion distributions of this wind turbine can be found in the bibliography ([18]).

As, for any operating condition, $M \ll 0.3$, the flow can be considered as incompressible. Also, the conservation equations are formulated in a MRF which moves with the angular velocity of the blade, $\Omega$. Formulating equations in such a reference frame allows to neglect transient effects and resolve only for a stationary mean flow. This last assumption is well suited as can be observed in the bibliography. For instance, Li et al. [38] performed dynamic simulations using Detached Eddy Simulation (DES) for the NREL phase VI. In this reference it can be found how, for an optimization purpose, the assumption of a stationary solution is well suited.

Other simplification which is to be made is the assumption of periodicity. Thus, a unique blade is being modeled, allowing to use a reduced domain with an angle $\Delta \theta=2 \pi / B$. This assumption has been successfully applied in the literature [39].

Thus the fluid domain is conformed by a truncated cone. The upwind distance is of $4 R$ while the downwind length is set to be $8 R$. The inlet radius is of $4 R$ and the outlet radius is $8 R$, where $R$ is the blade radius. These dimensions were set to ensure negligible influence of the position of the boundary conditions.

In order to ensure that the result is not dependent on the chosen discretization, the non-dimensional power and axial force curves are calculated for four different meshes. Figure 4 shows the power (left) and axial force (right) coefficients as they evolve with the increment of mesh elements, $N$. Note how both the power and axial curves achieve mesh independence with a relatively low number of elements at low values of $\lambda$ while, for $\lambda>5$, as the flow is attached to the blade, the necessary number of elements is higher. 

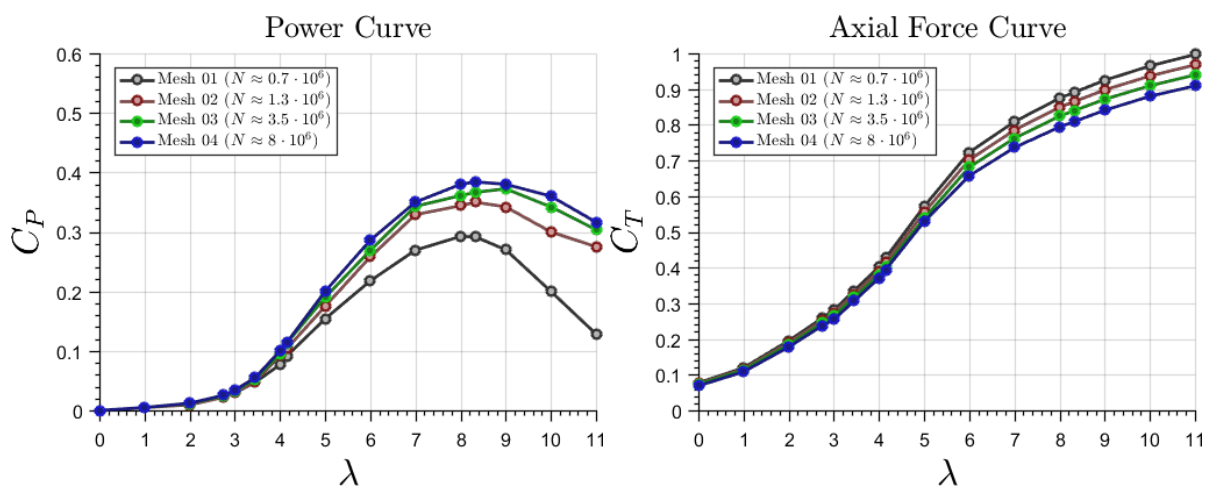

Figure 4: Power (left) and force (right) curves calculated for the blade NREL Phase VI using different grid refinements

Figure 4 allows to infer that, for fourth studied mesh, mesh independence can be considered to be achieved. In order to ensure reproducibility of results, note that this configuratoin corresponds to mean mesh size over the blade of approximately $0.005 R$ and a maximum size far from the blade of $0.25 R$. A correct flow resolution just upwind and at the wake is of great importance when computing forces and moments over the blade [40]. Thus, a mesh refinement zone is set in the wake, with a size of $0.02 R$, resulting in a polyhedral mesh with $N=8120000$ elements.

To obtain the non-dimensional power curve of the wind turbine a constant velocity inlet $V_{z}=8 \mathrm{~m} \mathrm{~s}^{-1}$ is set and the rotational velocity is parametrically varied. Under these circumstances the Reynolds number based on the relative tip velocity, $\operatorname{Re}=\rho R \sqrt{V_{z}^{2}+(\Omega R)^{2}} / \mu$, varies in the interval $\left[2 \cdot 10^{6}-6 \cdot 10^{6}\right]$ which is high enough to allow neglecting the effects of the Reynolds number on the friction coefficient.

The wall $y^{+}=y u_{\tau} / v$ was ensure to be greater than 30 for the major part of the studied geometry, so logarithmic wall functions can be used [41]. This can be ensured from the observation of the Figure 5, where the relative cells distribution of the wall $y^{+}$are shown for the cases of $\lambda=3$ (left) and $\lambda=6$ (right). Here, it can be calculated that, even for $\lambda=3$, more than the $70 \%$ of the wall cells show an $y^{+}$between 30 and 100 .
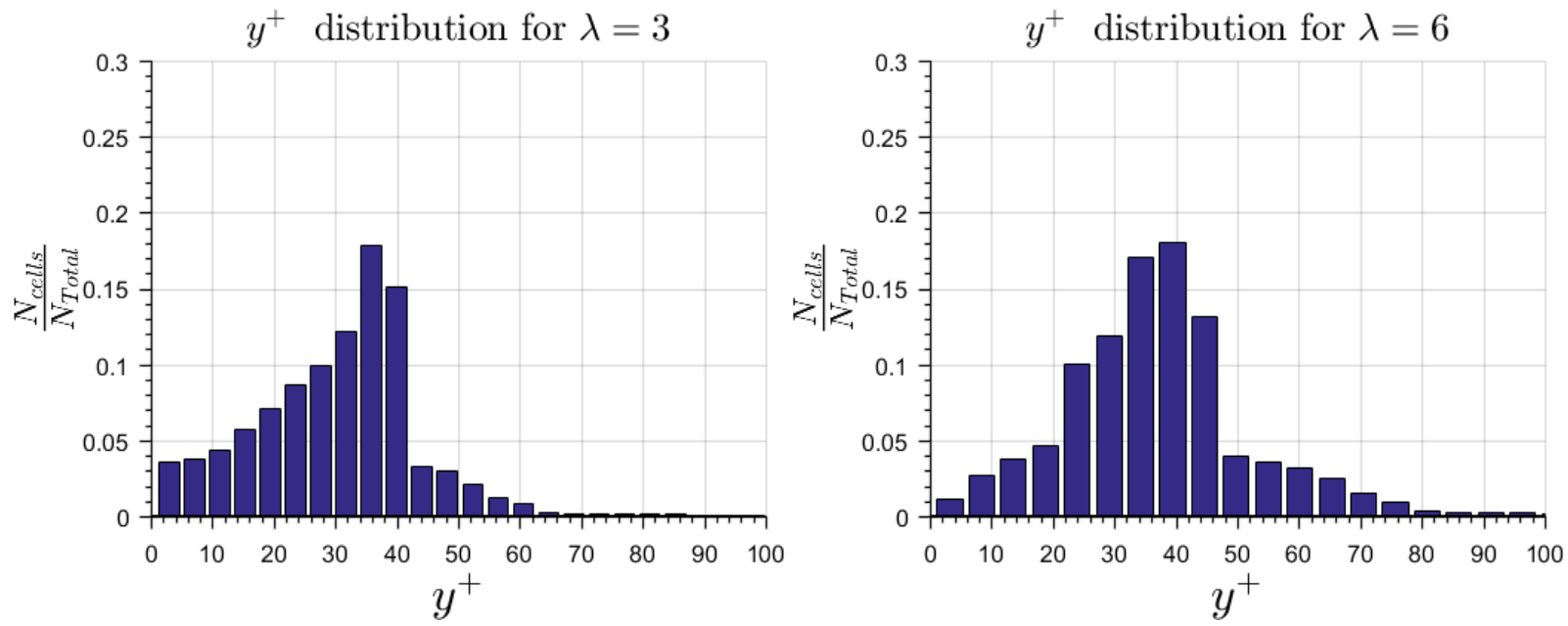

Figure 5: Wall $\mathrm{y}+$ distribution for $\lambda=3$ (left) and $\lambda=6$ (right). Calculations performed with the NREL Phase VI blade with $N \approx 8 \cdot 10^{6}$

Once the current numerical methodology was validated against the results of the NREL Phase VI bi-blade wind turbine (see section 3.3), the same specifications were applied to the optimum three-bladed low power wind turbine which will be the main target of the current article. As a result, a sketch of the mesh for this case can be found at Figure 6 where all the mentioned refinement levels can be observed at the same time that the angle of the truncated cone is found to be $\Delta \theta=120 \mathrm{deg}$, corresponding to $B=3$. 


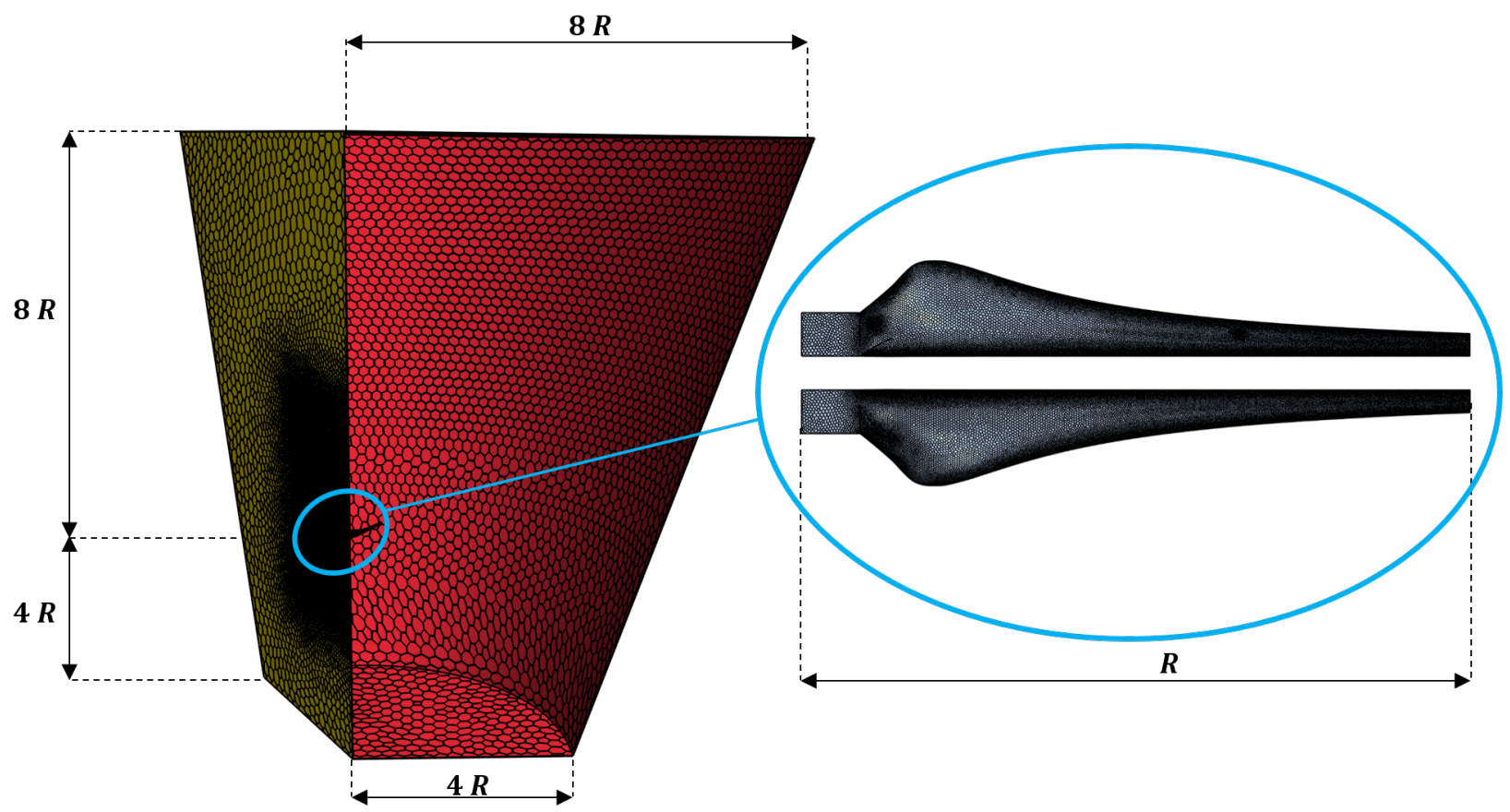

Figure 6: Mesh sketch for a case of a three bladed wind turbine calculation

\subsection{Model validation}

Both the BEMT and the CFD need to be validated against experimental data, for which the NREL Phase VI wind turbine was modeled in accordance with the conditions stated in the previous section. The selection of this two-bladed wind turbine was made due to the amount of work performed on it found in the literature ([42], [43]) and the available experimental data ([18], [19]).

Figure 7 shows the evolution of the pressure coefficient, $C_{p}$, along the blade chord for two different radial locations. The pressure is non-dimensionalized using the relative wind velocity at each section, as follows:

$$
C_{p}=\frac{p-p_{\infty}}{\frac{1}{2} \rho\left(V_{z}^{2}+(\Omega r)^{2}\right)}
$$

It can be observed that the current CFD calculations agree fairly well with the measurements. The agreement is slightly worse for low values of $\lambda$, for which the local angles of attack are larger [10]. Nevertheless, the results show good agreement even under these circumstances. 

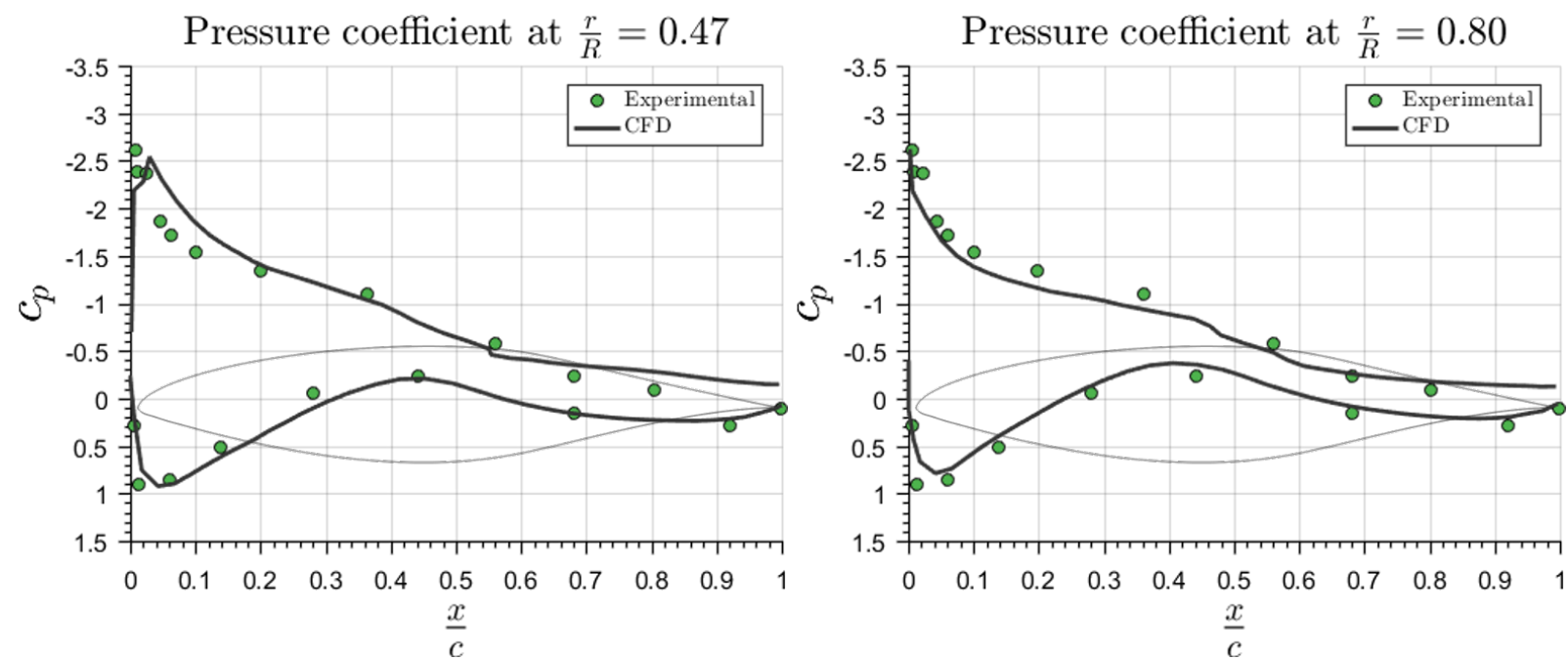

(a) Pressure coefficient for NREL Phase VI at $r / R=0.47$ (left) and $r / R=0.80$ (right) for $\lambda=6$
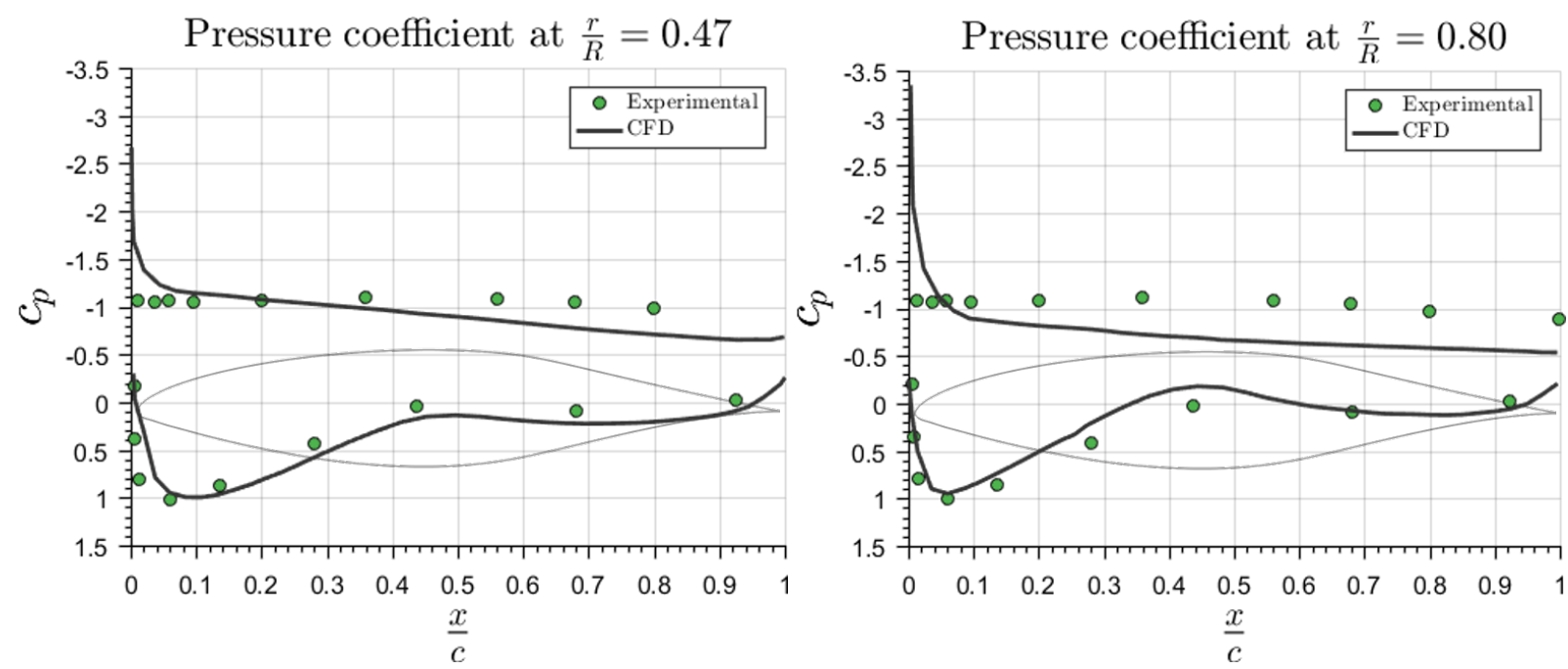

(b) Pressure coefficient for NREL Phase VI at $r / R=0.47$ (left) and $r / R=0.80$ (right) for $\lambda=2.78$

Figure 7: Pressure coefficient. Comparison between experiments and CFD

The evolution of the pressure coefficients that were shown in Figure 7 can be easily explained from the observation of the Figure 8, where the magnitude non-dimensional velocity $V^{*}=\frac{V}{\sqrt{(\Omega r)^{2}+V_{z}^{2}}}$ is shown in the rotating reference frame, in conjunction with the field streamlines, for the same characteristic airfoils which were use in the previous Figure. Note how, for the case with $\lambda=6$ the flow is attached for the blade, leading to a pressure distribution with a high pressure drop between pressure side and suction side. For $\lambda=3$ the flow is detached at these sections, leading to the almost constant pressure coefficient which can be observed at the suction side at Figure 7(b). 


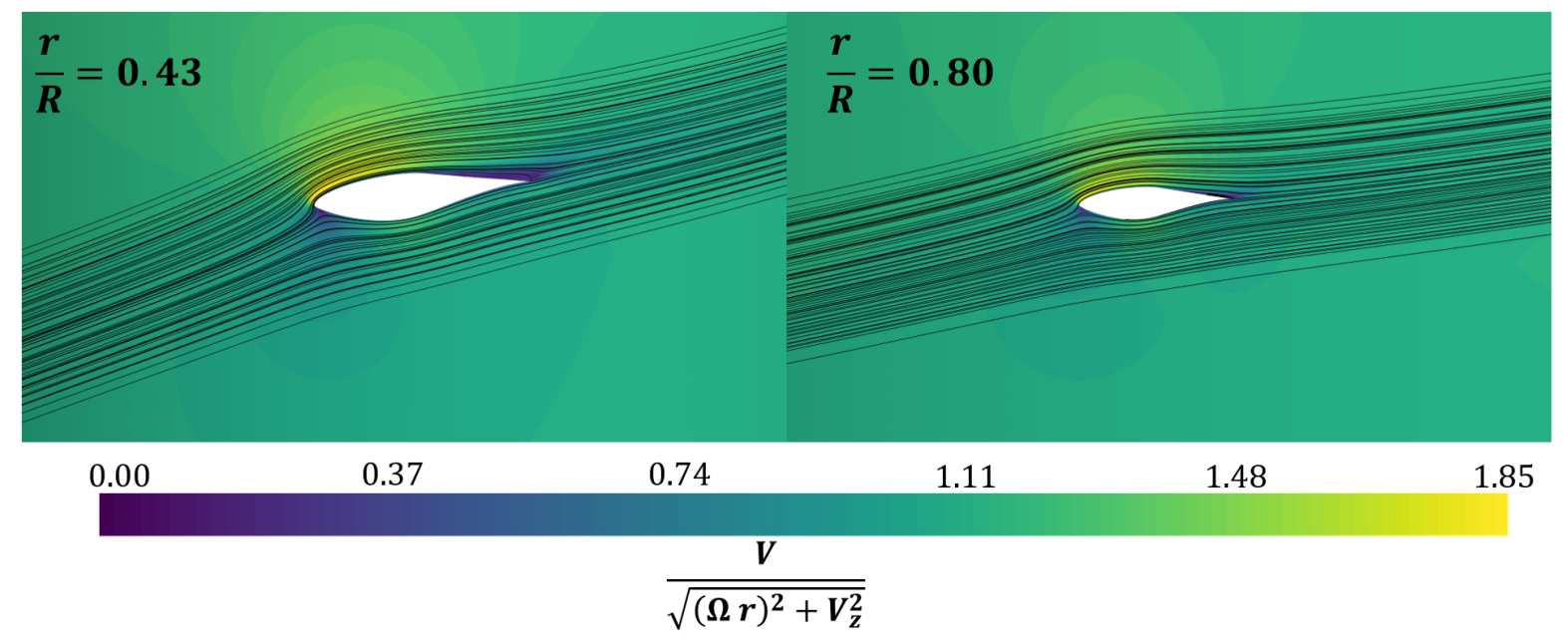

(a) Contours of non dimensional velocity field arround two different sections for $\lambda=6$

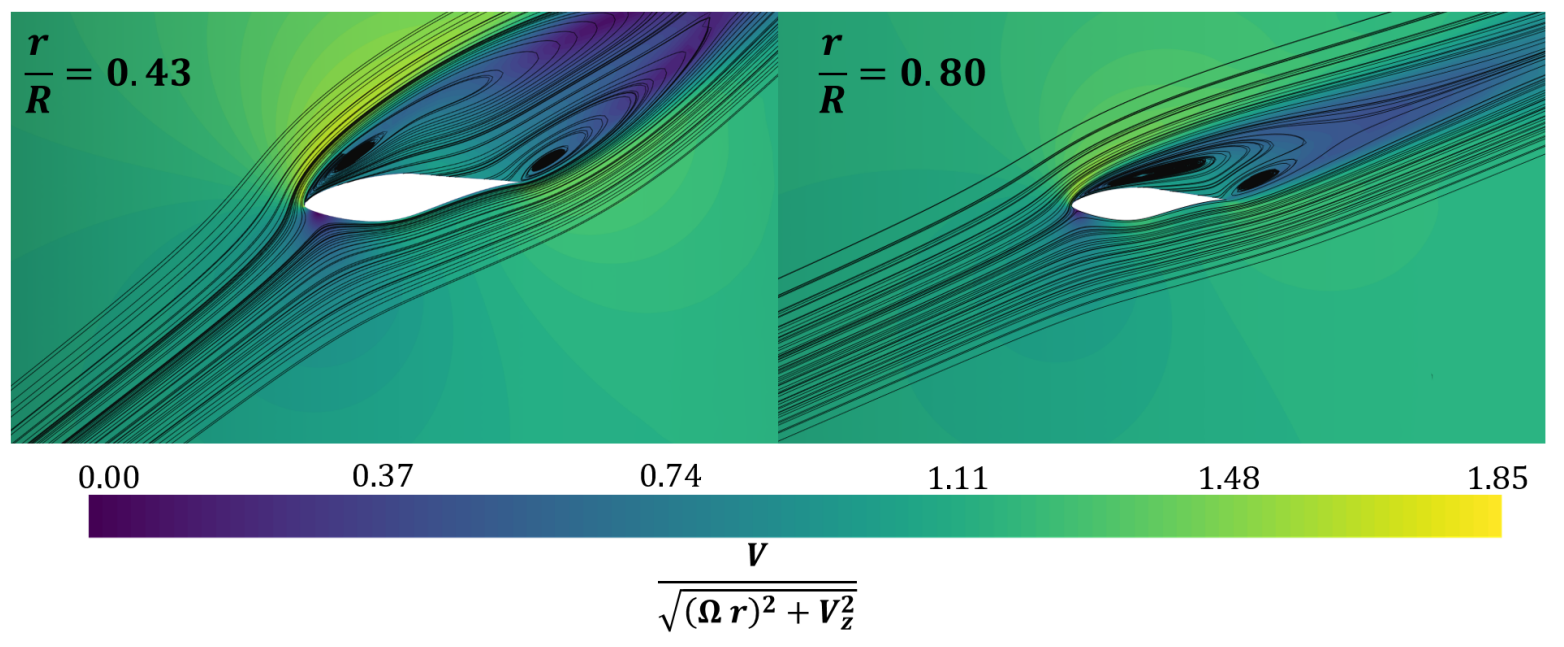

(b) Contours of non dimensional velocity field arround two different sections for $\lambda=2.78$

Figure 8: Velocity fields arround the NREL Phase VI geometry

Additionally, Figure 9 shows the value of the power coefficient as a function of $\lambda$. It can be observed that the current CFD-MRF simulations agree fairly with the experiments. As expected, and due to the limitations of the model, the BEMT tends to over-predict the power value for low values of $\lambda$. Nevertheless, this model is able to provide an acceptable preliminary prediction for the whole working range with a significantly reduced computation time. Thus, this model was used in order to perform a previous analysis of possible enhancement actions. However, it should be had into account that, if the new design's features lead to the appereance of important 3D effects, the BEMT-predicted power curve should be analyzed accounting for them. Thus, the BEMT-optimized wind turbine families were later studied using CFD in order to obtain a more realistic power curve. The comparison of the curves shows a difference of approximately a $3 \%$ on the prediction of the maximum power coefficient, and approximately a $12 \%$ on the location of the optimum tip speed ratio, which is in agreement with the already reported differences found by other researches [44] when comparing these methods. 


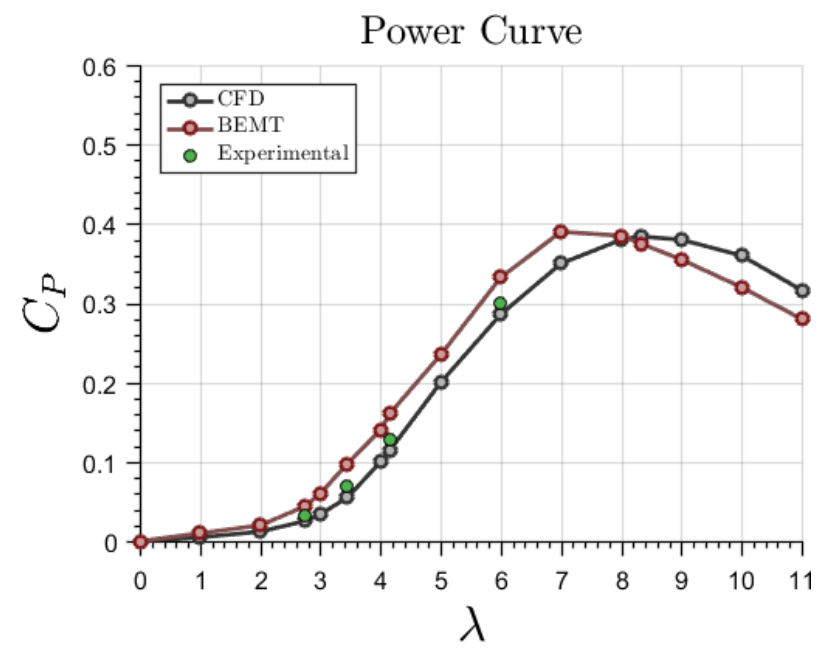

Figure 9: Power coefficient curve. Comparison between current methodologies and experiments

In order to check the validity of neglecting the influence of the Reynolds number the NREL Phase VI behavior curves have been calculated for three different sets of the velocity inlet, covering the whole expected working range. Figure 10 shows the power curve (left) and axial force (right) at $V_{z}=4 \mathrm{~ms}^{-1}, V_{z}=8 \mathrm{~ms}^{-1}, V_{z}=12 \mathrm{~ms}^{-1}$. It can be concluded that, although some differences can be found at high values of $\lambda$ they are below a 5\% and therefore, the assumption of Reynolds number independence can be considered to be accurate enough.

Power curve inlet velocity dependence

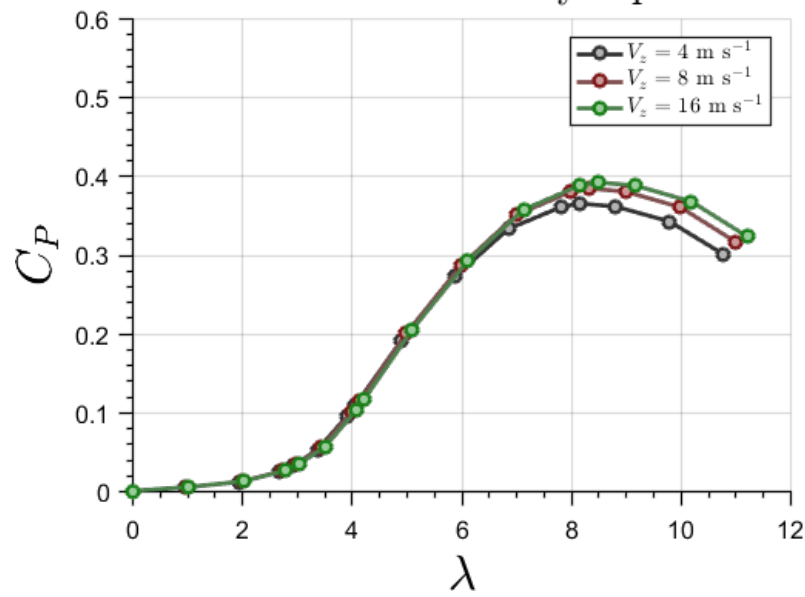

Axial force curve inlet velocity dependence

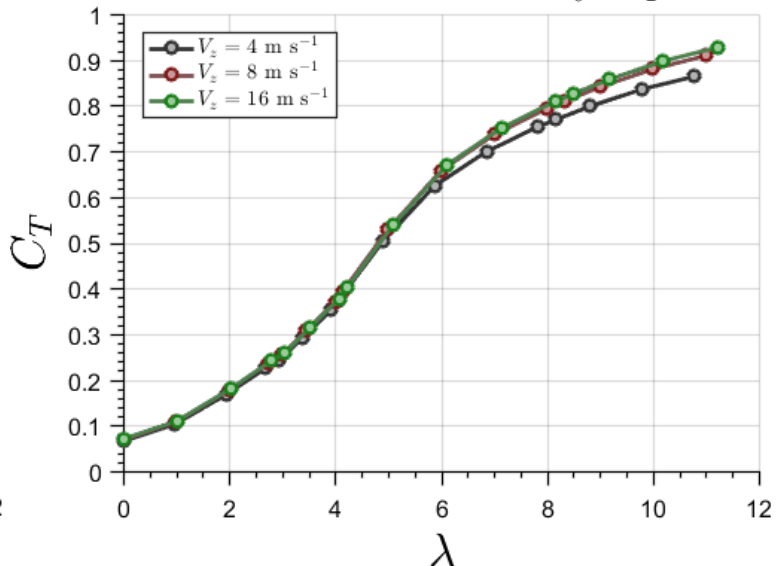

Figure 10: Power (left) and axial force (right) coefficient curves computed for the blade NREL Phase VI. CFD calculations at different inlet velocities

\section{Results}

\subsection{DOE results}

After the DOE was performed, a family of power curves was obtained. Figure 11 shows a typical selection of the different power optimization possibilities. As it can be observed, as $\lambda_{d}$ increases, the left slope of the curve is decreased. Also, it can be seen that when $\alpha_{d}$ is increased, the inverse trend is observed. During the design of experiments the number of blades was supposed to be $B=3$ and the radious was fixed to $R=2.50 \mathrm{~m}$.

For high values of $\alpha_{d}$ the power curve tends to values of $C_{p}<<1$ for low values of $\lambda$. From the point of view of maximum power coefficient and controllability (see Equation (11)), the designs shown for $\left(\lambda_{d}=4.5, \alpha_{d}=7 \mathrm{deg}\right.$ ) 
and $\left(\lambda_{d}=7.5, \alpha_{d}=7 \mathrm{deg}\right)$ could both be valid. Nevertheless, the manufacturing criterion is not complied with for the first combination of values, due to the high value of solidity that should be used. This can be seen at Figure 12, where these values are shown as a function of the non-dimensional distance to the hub. As a result, it was considered to select the case generated by $\left(\lambda_{d}=7.5, \alpha_{d}=7 \mathrm{deg}\right)$ from this set of blades.

The former explanation serves as an example in order to provide with a picture of how an automatic selection procedure allows a reduction of the blade designs, using a low number of starting blades with illustrative purposes. This very same procedure, applied to the whole 70 possible designs generated during the design of experiments explained in section 3.1 allowed a high reduction of the designs to be evaluated by means of CFD (a total of 5 blades were obtained in this case, although the number could be affected by the selection of the restrictions sketched in section 3.1).

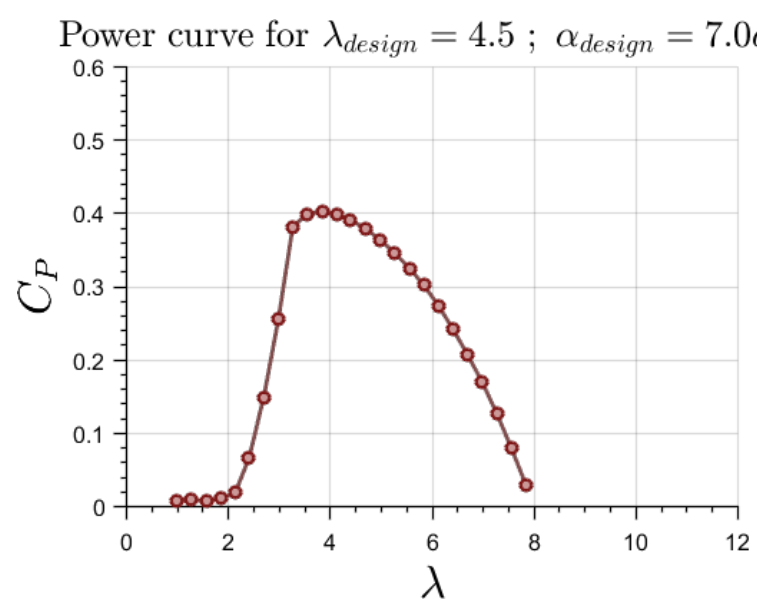

(a)

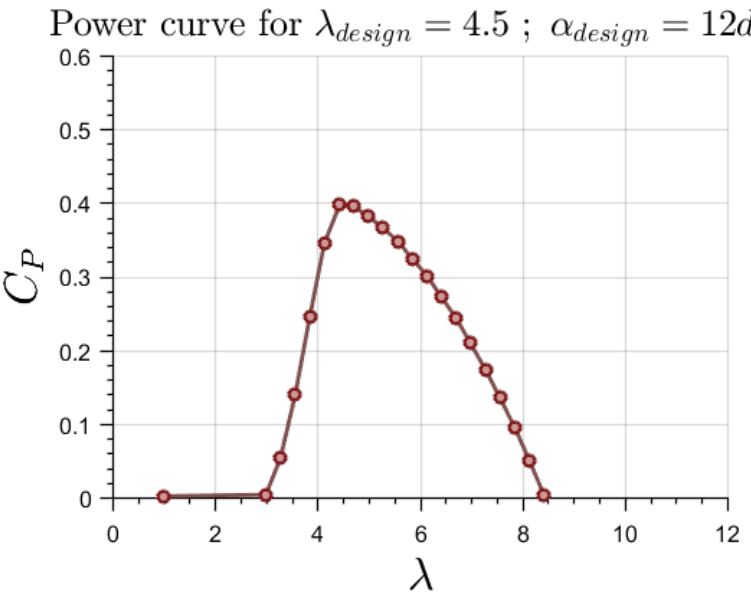

(c)

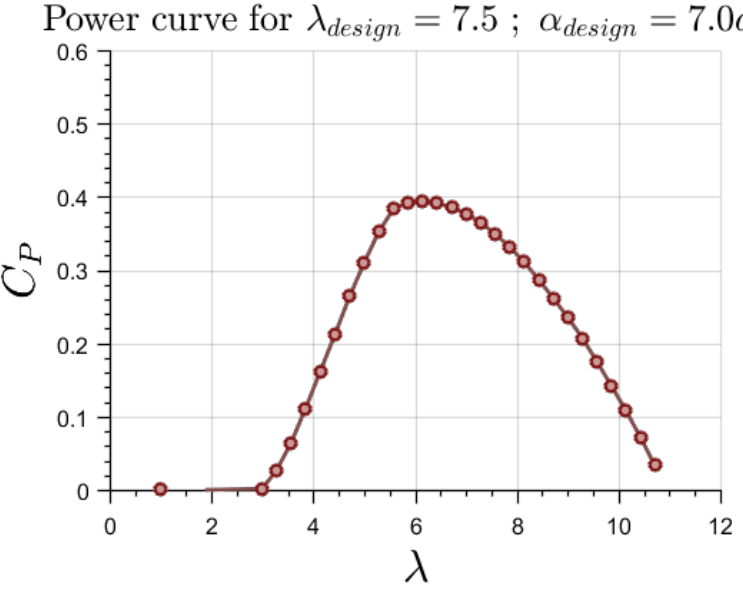

(b)

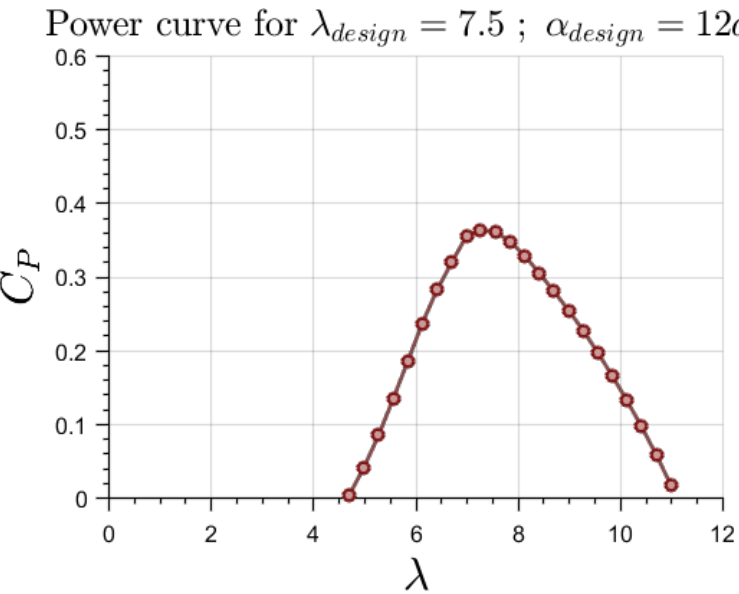

(d)

Figure 11: Power curves for different optimization parameters 


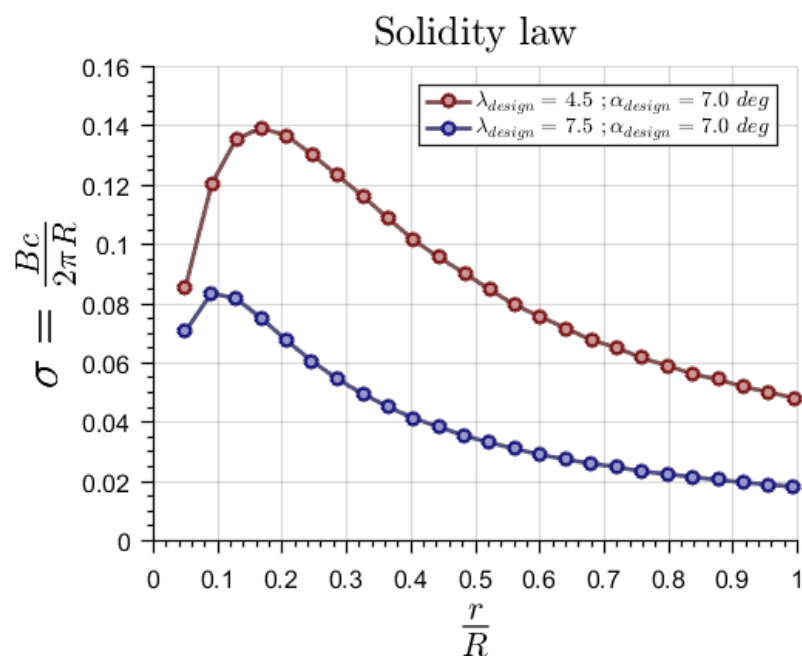

(a)

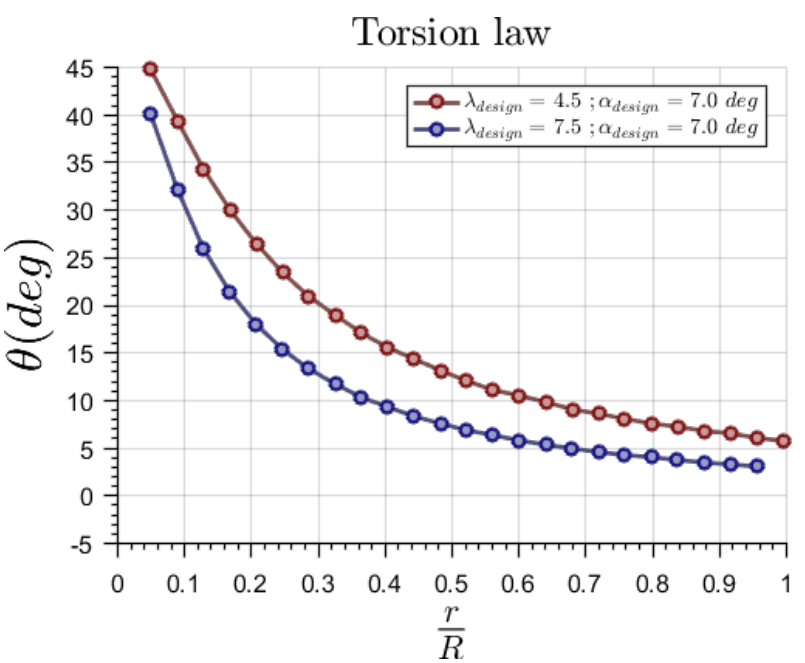

(b)

Figure 12: Local solidity (left) and torsion (right) distributions for two different resulting geometries from the DOE

The proposed blade design strategy was analyzed using the CFD model presented in subsection 3.2. The results on the power coefficient were compared by using both methodologies. Figure 13 (left) shows the comparison between the predictions which were made during the design process using BEMT and the 3D CFD steady calculations. It can be observed that the major discrepancies appear in the prediction of the location of the tip speed ratio of maximum power coefficient, where a difference of the order of $11 \%$ is obtained. The value of the maximum $C_{P}$ is well predicted, with a difference of approximately a $4 \%$. Similar differences between CFD and BEMT have already been reported by other researches applied to the MEXICO rotor aerodynamics [44].

A visual inspection of the power curve shown in Figure 13 (left) allows to observe how the qualitative differences at low values of $\lambda$ could look to be more accentuated than those observed during the validation study of Figure 9 . This is mainly due to the narrow shape of the power curve for the case of the current proposed blade design. Although the differences on the location of the maximum power coefficient and the maximum coefficient itself are on a similar order of those which where shown in Section 3.3, the high left slope of the curve makes them more visible.
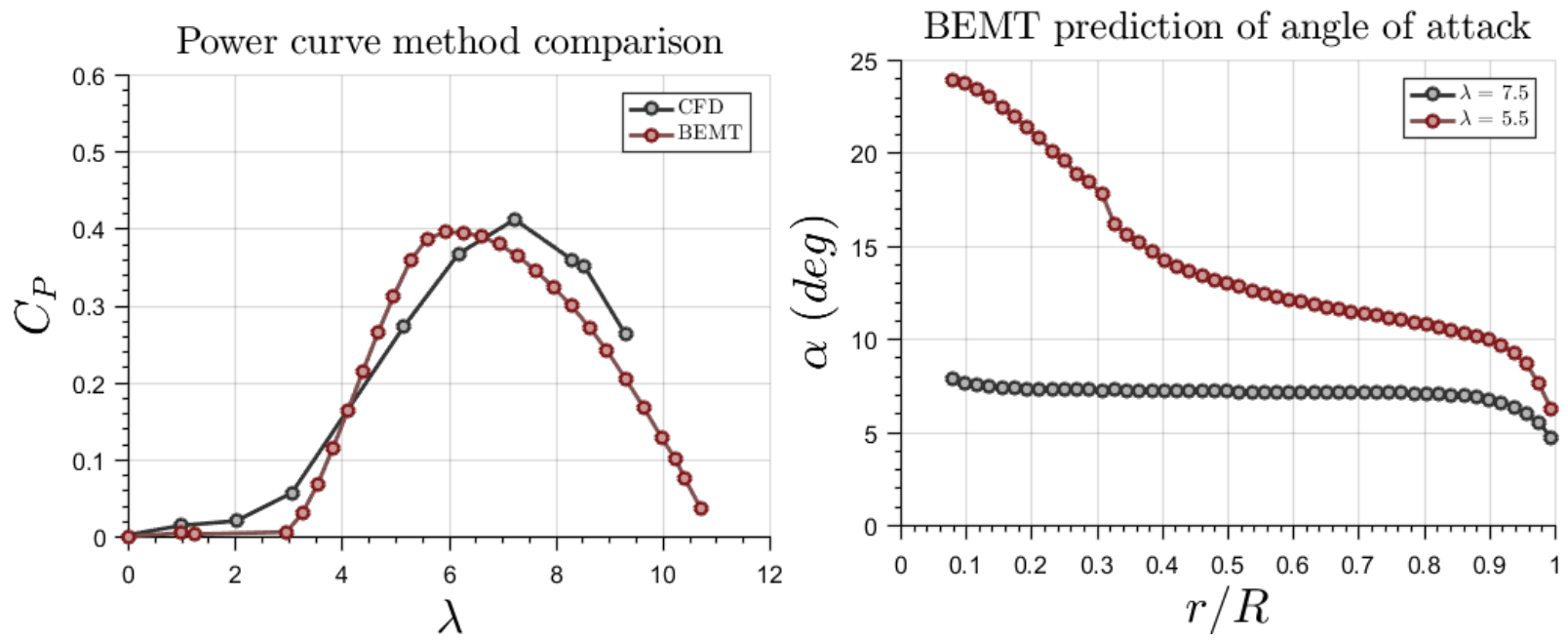

Figure 13: Comparison between the CFD and BEMT predicted power curves for the current proposed geometry (left) and BEMT prediction of the angle of attack for two different tip speed ratio (right) 
Also, it can be observed that, for low values of the tip speed ratio $(\lambda \in[4-5])$, the BEMT tends to over-predict the power coefficient, which is mainly due to the non-inclusion of the 3D stall delay phenomenon in the model.

Additionally, it can be seen that the left side slope of the power coefficient curve is over-predicted by the BEMT. However, this discrepancy is compensated by the increase of $\lambda$, from the controllability point of view, in accordance with Equation (11). Thus, the BEMT can be considered to be sufficiently good for the DOE performed, as will be later shown.

The differences on the prediction of the power coefficient for low values of $\lambda$ (and, as a consequence, on the prediction of the power curve slope) can be interpreted referring to Figure 13 (right). In this Figure, the BEMT predicted angle of attack is compared for two different values of the tip speed ratio: For a value of $\lambda=7.5=\lambda_{\text {design }}$ and $\lambda=5.5<\lambda_{\text {design }}$. Note how, for the former, an almost constant value of the angle of attack $\alpha \approx 7.0$ deg $=\alpha_{\text {design }}$ is found for the most part of the blade. When the value of $\lambda$ decreases, as expected, the predicted angle of attack tends to increase. Due to the high difference on torsion angle between the root and the tip, compared with NREL Phase VI, this increment on angle of attack is more abrupt at the root. This, generates an important gradient of angle of attack under these conditions which, in conjunction with being working at stall conditions, will lead to the appeareance of very important $3 \mathrm{D}$ effects which are not taken into account with the current BEMT.

It can also be noted how, for these geometries, if no additional three dimensional corrections are applied to the BEMT, the predictions at very low values of the tip speed ratio $(\lambda \leq 3)$ can not be considered to be sufficiently accurate, and therefore, CFD or experimental measurements should be used in order to obtain valuable results at these conditions.

\subsection{Comparison against other designs}

As it was previously mentioned, once a design is generated and selected via the Design of Experiments, its behavior must be studied and analyzed against a functioning known design. The blade of the low power wind turbine Skystream was selected in order to do so, as this model is one of the current leaders in the market of low wind power generation. The geometry of the commercial blade was digitalized, so CFD calculations can be performed. Note also that, as stated before, due to the horizontal curvature of the blade, the current BEMT methodology should no directly be applied for this problem. Figure 14 shows a geometrical comparison between the proposed current design (left) and the Skystream geometry (middle). Figure 14 (right) shows a zoom of the Skystream geometry, where a geometrical protuberance can be observed for most of the radial coordinates of the blade.

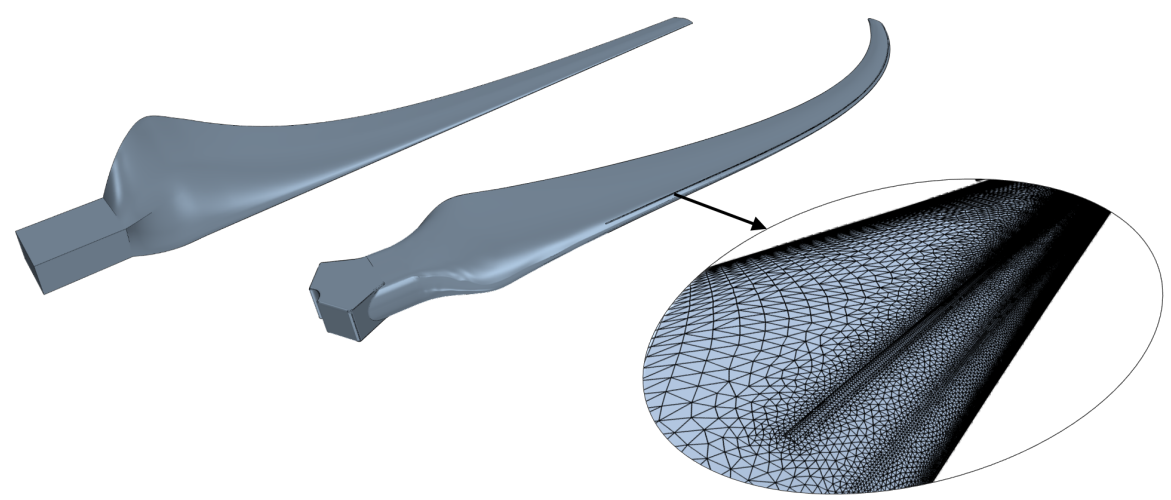

Figure 14: Comparison between current design (left) and a commercial geometry (right)

The mentioned geometrical feature will act as a Vortex Generator (VG) and its aim would be influencing on the boundary layer behavior. Due to the usual turbulence intensities and three dimensional flows this kind of products are subjected to, it is expected that setting the transition between a laminar and a turbulent boundary layer will not be 
primal. Then it is necessary to study how the VG will affect to the energy production, and the Skystream geometry with the VG removed was also be studied.

With this aim, Figure 15 shows the CFD-calculated power curve for the mentioned geometries. As it can be seen, the current design shows higher values of the power coefficient for a wide range near the working zone (from $\lambda=6.2$ to $\lambda=8.5$. For $\lambda<6.2$ the current design shows a pronounced slope and lower $C_{P}$ values, contributing to a better stability for high values of $V_{z}$. Additionally, the peak value of $C_{P}$ is improved up to a $14 \%$ compared with the commercial design.

It is also of interest the comparison between the commercial design with and without the VG. The design with VG shows a lower value of $C_{P}$ for the whole working range of the wind turbine. This allows to state that seems that this VG does not improve the wind turbine performance from the point of view of energy generation. However, the reduction of power coefficient on the whole range could lead to a better stability performance.

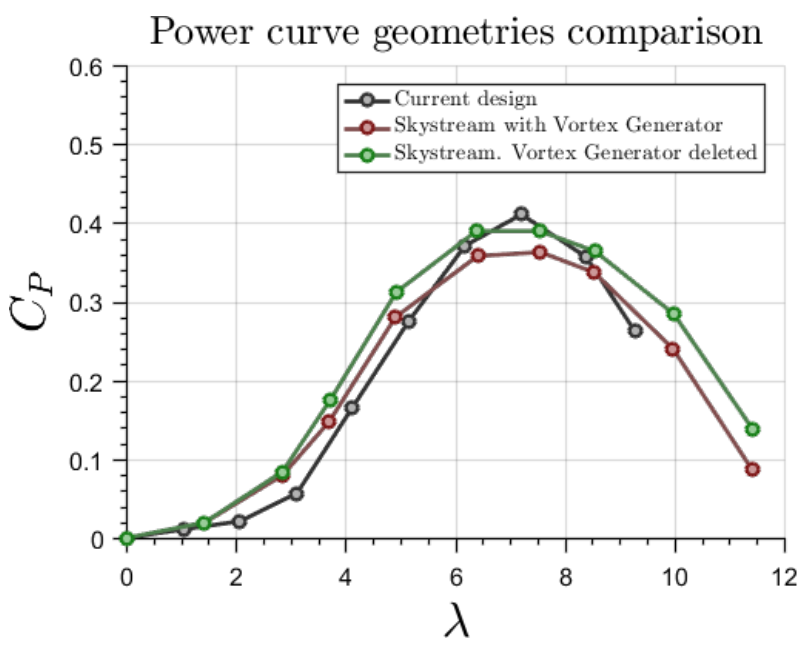

Figure 15: Comparison of the power curve for the different analyzed blades

The differences in the pressure coefficient between the different geometries are closely related to the pressure distribution over the suction side and the pressure side of the different blades at different operating conditions. Figure 16 shows the pressure coefficient distribution, defined in accordance with Equation (13). For each blade the suction side and the pressure side are shown above and below, respectively. Notice that the pressure coefficient presents higher values over the pressure side in all the cases. For values of $\lambda \approx \lambda_{\text {opt }}$ the pressure coefficient over the suction side exhibits a distinct minimum close to the blade leading edge.

In Figure 16 lines which are tangent to the wall shear stress at each blade point are represented. The locations where these lines present abrupt changes represent the zones where the flow is suddenly separated. As a general trend for all the designs it can be observed that, for low values of $\lambda$, the area where the flow is separated tends to be increased. This is due to the higher local angles of attack that appear at these low rotational velocities (see Equation (1)).

Regarding the comparison between the different design solutions it can be observed that, for the current enhanced design (Figure 16(a) and 16(b)), the blade surface covered by the separation area is smaller than in the case of the commercial blade (Figure 16(c) and 16(d)). The removal of the VG in the commercial blade (Figures 16(d) and 16(e)) significantly reduces the separation area, specially for high values of the tip speed ratio. This phenomenon is strongly related to the fact that the blade without VG tends to produce a higher power, specially for high values of $\lambda$. 


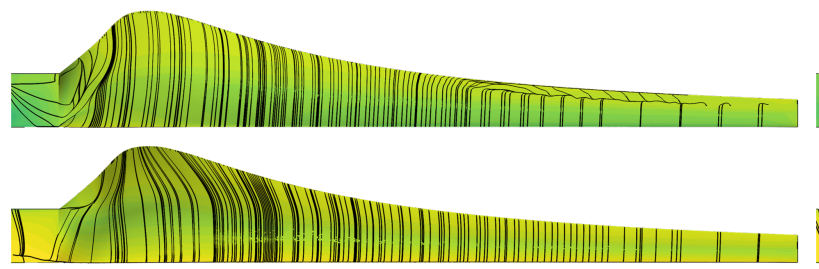

(a) Current enhanced design $\lambda=\lambda_{\text {optim }}$

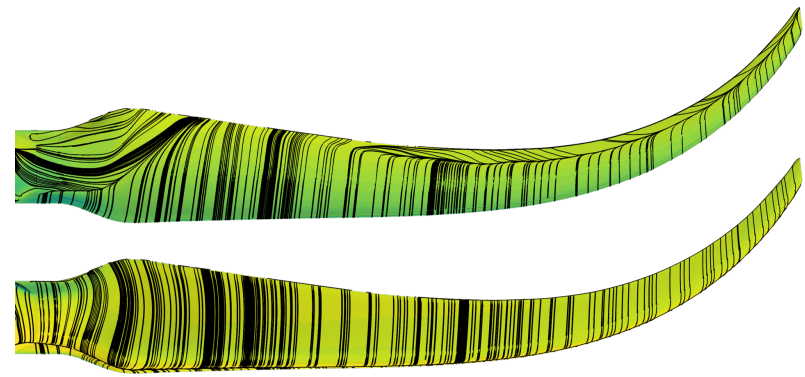

(c) Commercial blade $\lambda=\lambda_{\text {optim }}$

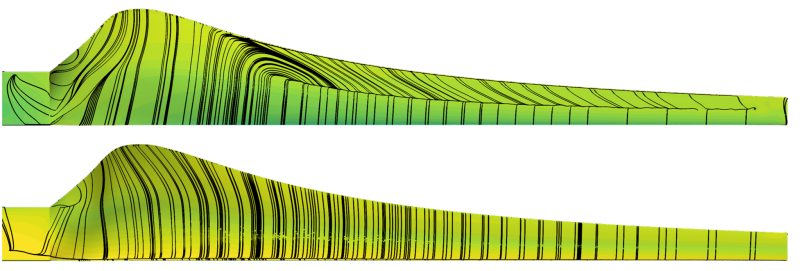

(b) Current enhanced design $\lambda \ll \lambda_{\text {optim }}$

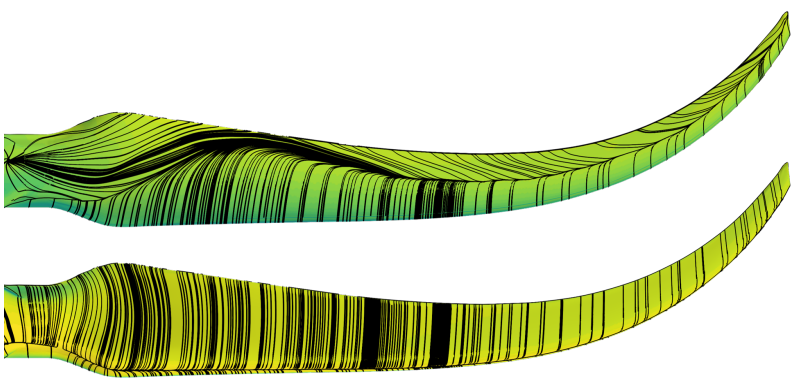

(d) Commercial blade $\lambda \ll \lambda_{\text {optim }}$

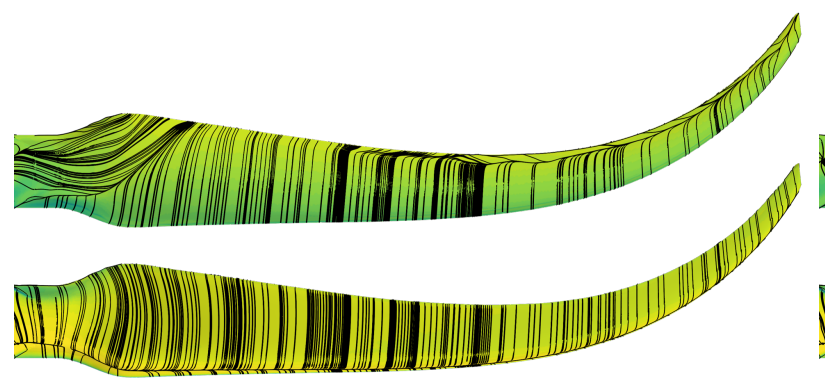

(e) Commercial blade w/o VG $\lambda=\lambda_{\text {optim }}$

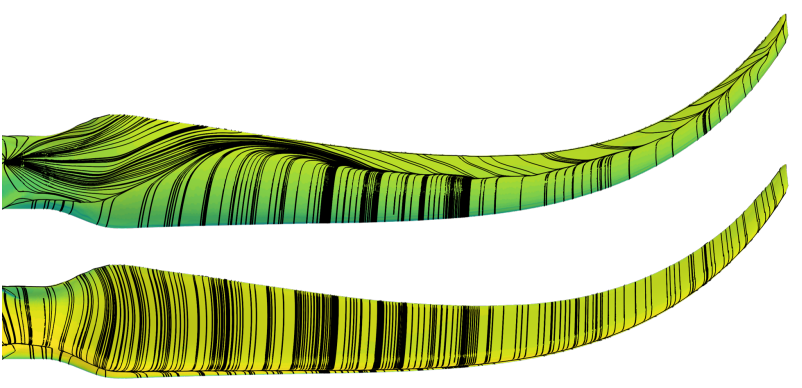

(f) Commercial blade w/o VG $\lambda \ll \lambda_{\text {optim }}$

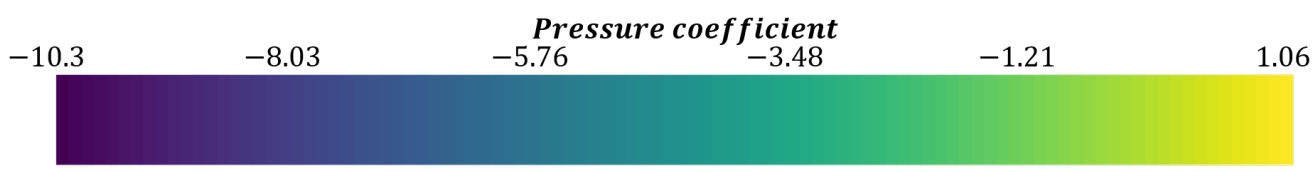

Figure 16: Contours of the pressure coefficient for the current design (up); the Skystream commercial blade (middle) and the Skystream commercial blade with the VG removed at the value of tip speed ratio of maximum power (left) and a low tip speed ratio (right) and stream lines of wall shear stress.

Once the non dimensional behavior of the proposed current design and the commercial blade has been analyzed, it is necessary to perform a validation on the controllability parameter expressed by the left-slope condition of Equation 11. Additionally, it will be shown the necessity of performing a CFD analysis to correct BEMT predictions of other candidate geometries in order to discard those which are expected to present difficult controllability issues.

As it was previously mentioned, during the development of the Design of Experiments multiple candidate geometries were obtained. In particular, additionally to the already mentioned proposed design (which it should be remembered was generated by using the parameters $\lambda_{d}=7.5$ and $\alpha_{d}=7 \mathrm{deg}$ ), other geometries were considered. One example could be the design which can be reconstructed by an application of the parameters $\lambda_{d}=6.5$ and $\alpha_{d}=6 \mathrm{deg}$.

The non dimensional power curve (left) and non dimensional torque curve $\left(C_{Q}=C_{P} / \lambda\right)$ (right) of this alternative design are shown in comparison with the corresponding curves of the current design and the commercial blade at Figure 17. Observe how this alternative design shows a power coefficient slightly higher than the shown by the 
proposed blade. Furthermore, note how the left slope of the power coefficient is significantly lower. Intuitively, one could assign low slopes of the power coefficient to easier control but, due to the cubic dependence of the dimensional power with incoming fluid velocity (see the derivation of Equation 11) this is not the case and, therefore, it should be ensured that, when applying a mechanical brake under high wind conditions, the power coefficient (and, therefore, the torque coefficient) should quickly decrease to compensate the increment of velocity.

This fact can be easily understood by a numerical application. Once the non dimensional power and torque coefficients are obtained in terms of the tip speed ratio, and given that its dependence with Reynolds number is of second order (see Figure 10), it is straightforward to obtain the dimensional power, given a wind turbine radius, for any combination of rotational speed $\Omega$ and wind velocity, $V_{z}$.

One typical law of control which is often followed by stall regulated wind turbines is to work on conditions of maximum power coefficient, $\lambda=\lambda_{\text {opt }}$, for low values of wind speed and with constant rotational speed $\Omega=\Omega_{\text {max }}$ when wind velocity exceeds certain value [45], [46], [47]. When the wind velocity becomes even higher, a brake torque must be employed in order to stop the wind turbine. In order to ensure that this is possible it is mandatory that both the generated power and torque do not increase with wind velocity in an uncontrolled manner.

Therefore, Figure 18 shows the prediction of the dimensional power and torque, calculated for the blades of Figure 17 , supposing that they are working under a normal ambient with $\rho_{\infty}=1.225 \mathrm{~kg} \mathrm{~m}^{-3}$. They will be supposed to be of the same size, with $R=2.50 \mathrm{~m}$ and they will work on conditions of maximum power coefficient, $\lambda_{\text {optim }}$ until the rotational speed exceeds a value of $\Omega_{\max }=170 \mathrm{rpm}$. For higher values of the velocity the rotational speed is maintained and, as a consequence, the value of $\lambda$ will be decreasing.

It can be observed how, for values of the wind velocity below $V_{z}=8 \mathrm{~m} \mathrm{~s}^{-1}$ the three blades behave in a very similar manner in terms of power and torque. However, when the wind speed increases the power of the discarded design increases in an uncontrollable manner. This can be attributed to the low value of the left-slope, which relates with a high value of the torque coefficient. Similar tend can be observed at the commercial blade, which shows the same behavior, although slower. The proposed design shows how, when wind speed exceeds from $V_{z}=12 \mathrm{~m} \mathrm{~s}^{-1}$ both the power and torque stay bounded and, therefore, it will be possible to stop the wind turbine.

Power curve

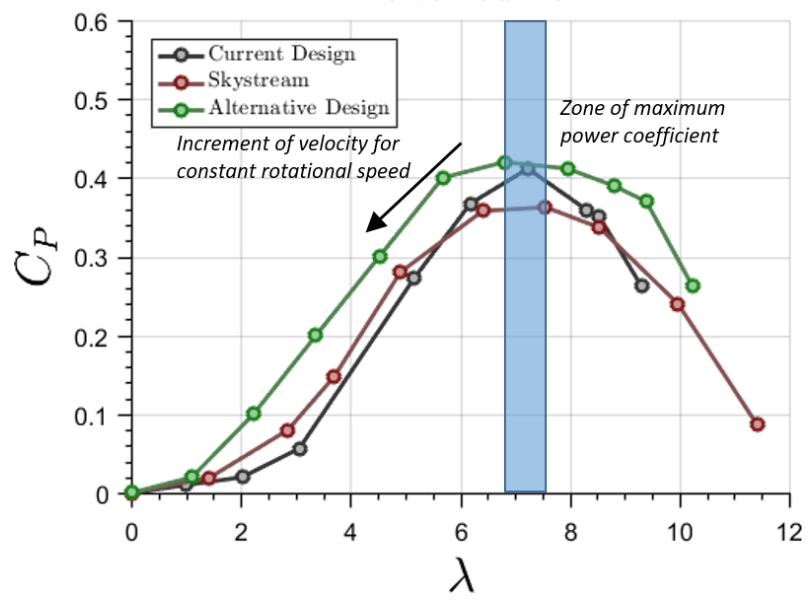

Torque Curve

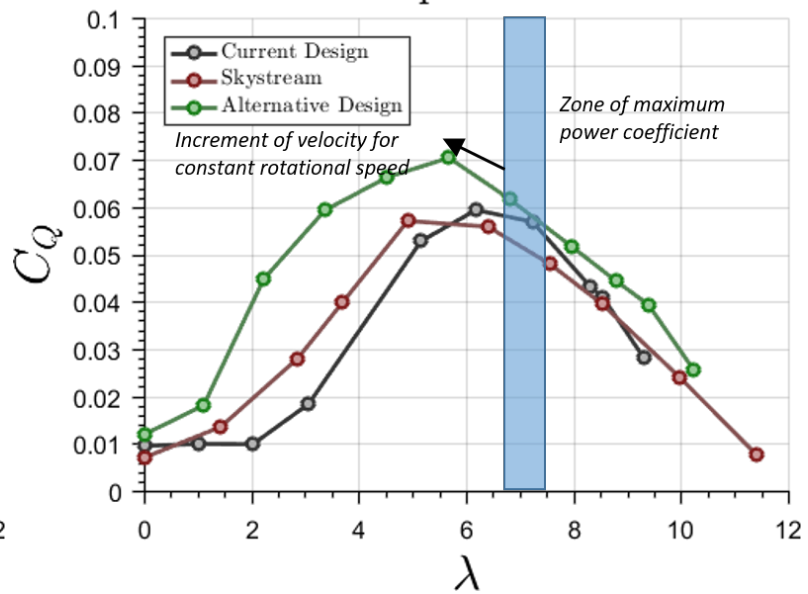

Figure 17: Evolution of both non dimensional power and torque for the current design (gray), the commercial blade (red) and an alternative discarded design (green). Predictions made with RANS 

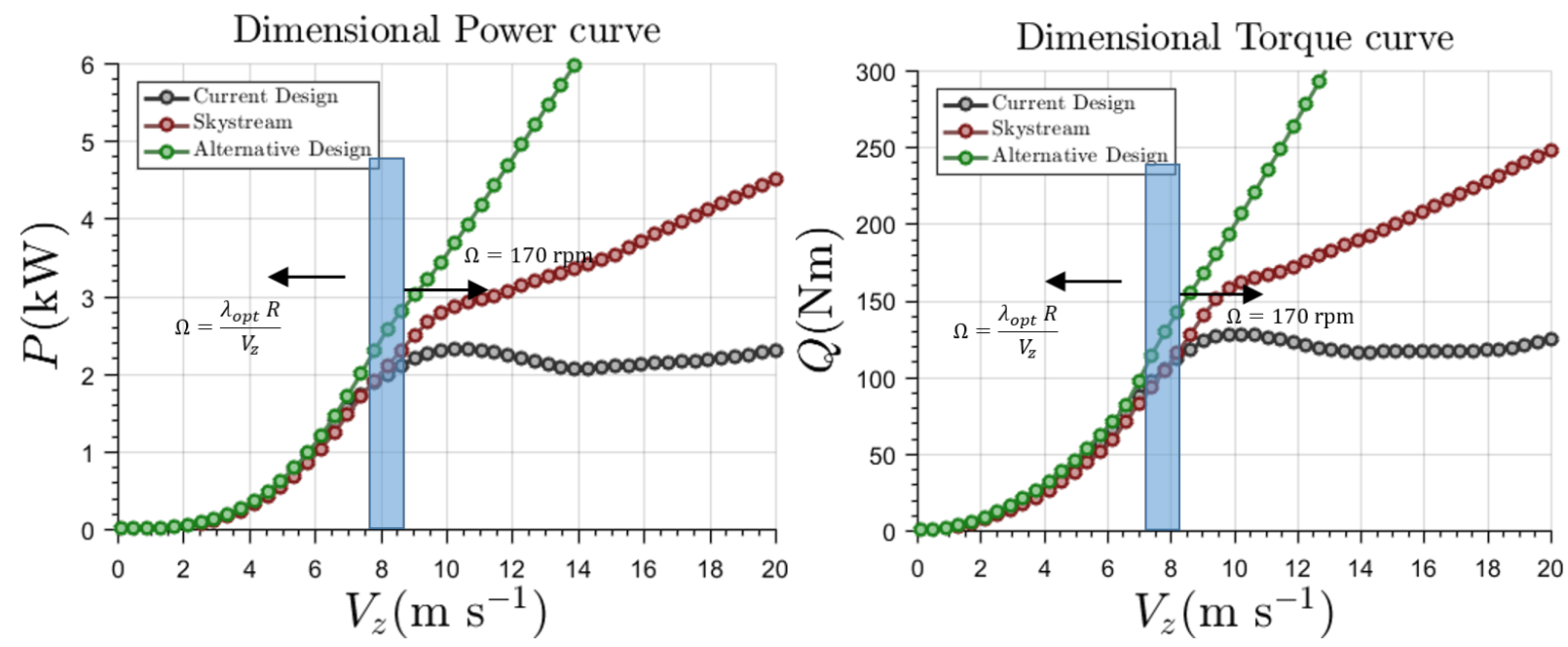

Figure 18: Evolution of both dimensional power and torque for the current design (gray), the commercial blade (red) and an alternative discarded design (green) with wind velocity. Numerical application supposing wind turbines of similar radius $R=2.50 \mathrm{~m}$ working under normal conditions with $\rho_{\infty}=1.225 \mathrm{~kg} \mathrm{~m}^{-3}$

Previous statements refer just to a direct application with the data obtained in this work. Nevertheless, conclusions may be extended to other control laws. Figure 19 shows the performance of a wind-turbine equipped with the alternative design by using different control laws at different maximum rotational velocity (50 rpm, $100 \mathrm{rpm}$ and $200 \mathrm{rpm}$ ). It could be stated that a completely controllable torque of the wind turbine is obtained at a lower rotational velocity values $(50 \mathrm{rpm})$ despite both the torque and power curves are monotonically increasing. However, this is achieved by drastically decreasing the generated power, since the wind turbine is always working far from the optimum value of $\lambda$. If the rotational speed is increased to $100 \mathrm{rpm}$, admissible power around $2.5 \mathrm{~kW}$ could only be achieved just with wind velocities above $20 \mathrm{~m} \mathrm{~s}^{-1}$.

Power curve

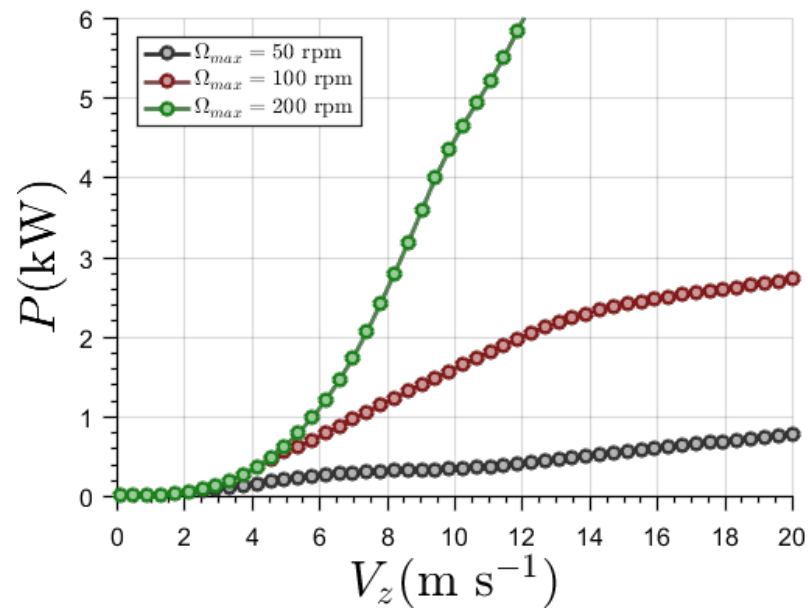

Torque curve

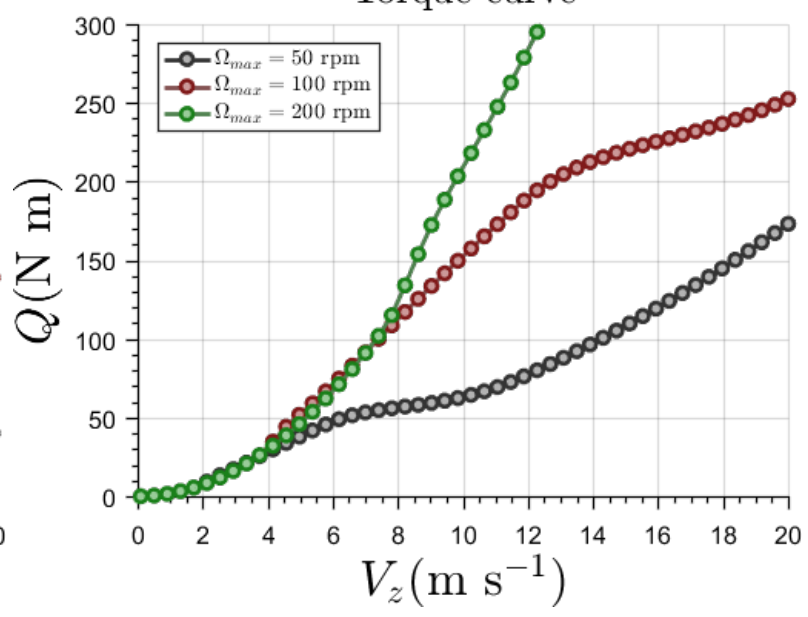

Figure 19: Evolution of the power (left) and torque (right) of the discarded design as a function of maximum control rotational velocity for a given radius of $R=2.50 \mathrm{~m}$

\section{Conclusions}

The current work provides with a wind turbine blade design methodology which can be considered as a flexible accurate tool. The method can be used as the base for the development of designs of increased complexity, given 
that the work has been presented with a complete validation and discussion of the differences between the used tools. For instance, the methodology can be used in order to explore the sensitivity of the restrictions, adding or removing them as a function of the design objectives or even to propose an optimization tool based on the minimization of an objective function based on the current proposed restrictions. Particularly, it has been applied to the design of a low power stall regulated wind turbine.

The methodology combines a complete set of design of experiments using a low computational cost tool as BEMT in order to select blade geometries for optimal performance and control. Furthermore, CFD methodology has been presented to accurately take into account three dimensional flow effects and, therefore, allow to discard those geometries which were initially valid in accordance with BEMT but are not easily controllable once these three dimensional effects are taken into account.

This combined methodology has been validated with experimental results available from NREL Phase VI wind turbine public data catalog. Results have been successfully compared in terms of wind turbine power but also in terms of pressure coefficients along the complete blade at different radial sections.

The proposed BEMT design procedure includes additional restrictions to the power curve form, in order to obtain a blade geometry which, not only maximizes the power coefficient, but allows having into account controllable issues for the design of stall regulated HAWT. As a result, a reduced set of feasible blade designs has been obtained, allowing to perform CFD calculations over them and finally select a blade which allows both an increment in maximum power coefficient (compared with a commercial design) and an almost flat dimensional power curve for a simple control law.

The predicted behavior of the proposed design has been analyzed in terms of power generation and controllability and compared to the predictions over a commercial existing wind turbine under similar working conditions. This comparison showed how the new design allows obtaining higher values of power while improving the velocity range at which the turbine could safely operate.

Additionally, the influence of Vortex Generators, installed near the blade leading edge, on the performance and control ability has been also studied. It was found that VG solution proposed seems to negatively affects maximum power generation. Furthermore, VGs affects flow separation phenomena and therefore the capability of the blade to control the rotation velocity of the wind turbine.

It has been shown how a combination of the Blade Element Momentum Theory and Reynolds Averaged Navier Stokes can be used in order to take advantage of the capabilities which are related with each model:

- BEMT allowed to obtain a significant amount of candidate designs at low CPU cost, and discard those candidates that clearly do not comply with the performance requirements. Nevertheless, it does not take in to account 3D effects which could be important for the correct prediction of performance at low TSR values.

- RANS has been used in this work to accurately obtain behavior of a reduced number of pre-selected designs and to discard those not fulfilling the performance and control requirements.

Finally, the left-slope of the non-dimensional power curve has been shown to be a key parameter for the successful design of a stall regulated wind turbine. In fact, taking into consideration this parameter as a relatively simple design constraint allows one to generate a blade geometry which is able to provide higher power than similar designs and can safely operate in a wide range of wind velocities.

\section{References}

[1] M. Jacobson, M. Delucchi, Providing all global energy with wind, water, and solar power, Part I: Technologies, energy resources, quantities and areas of infrastructure, and materials, Energy Policy 39 (2011) 1154-1169.

[2] S. Schreck, Wind Annex XX: HAWT Aerodynamics Models from Wind Tunnel Measurements, Tech. Rep., National Renewable Energy Laboratory, 2008

[3] T. Muche, R. Pohl, C. Hge, Economically optimal configuration of onshore horizontal axis wind turbines, Renewable Energy 90 (2016) $469-480$.

[4] IEC61400-2, Wind Turbines. Part 2-design requirements for small turbines, Tech. Rep., 2006.

[5] S. Roshan, S. Alimirazadeh, M. Rad, RANS simulation of the stepped duct effect on the performance of ducted wind turbine, Journal of Wind Engineering and Industrial Aerodynamics 145 (2015) 270-279.

[6] M. Lee, Y. Shiah, C. Bai, Experiments and numerical simulations of the rotor-blade performance for a small-scale horizontal axis wind turbine, Journal of Wind Engineering and Industrial Aerodynamics 149 (2016) 17-29. 
[7] E. Benini, A. Toffolo, Optimal design of horizontal-axis wind turbines using blade-element theory and evolutionary computation, Journal of solar energy engineering 124 (2002) 357-363.

[8] R. Rooij, E. Arens, Analysis of the experimental and computational flow characteristics with respect to the augmented lift phenomenon caused by blade rotation, Journal of Physics: Conference Series 75 (2007) 012021.

[9] Z. Du, M. Selig, A 3-D stall-delay model for horizontal axis wind turbine performance prediction, AIAA paper 21 (1998) 9-19.

[10] J. Manwell, J. McGowan, A. Rogers, Wind Energy Explained, Wiley, 2009.

[11] J. Mo, Y. Lee, CFD Investigation on the aerodynamic characteristics of a small-sized wind turbine of NREL Phase VI operating with a stall-regulated method, Journal of Mechanical Science and Technology 26 (2012) 81-92.

[12] T. Tran, D. Kim, A CFD study into the influence of unsteady aerodynamic interference on wind turbine surge motion, Renewable Energy 90 (2016) 204-228.

[13] J. Mo, A. Choudhry, M. Arjomandi, Y. Lee, Large eddy simulation of the wind turbine wake characteristics in the numerical wind tunnel model, Journal of Wind Engineering and Industrial Aerodynamics 112 (2013) 11-24.

[14] S. Kang, X. Yang, F. Sotiropoulis, On the onset of wake meandering for an axial flow turbine in a turbulent open channel flow, Journal of Fluid Mechanics 744 (2014) 376-403.

[15] C. Santoni, J. Carrasquillo, I. Arenas-Navarro, S. Leonardi, Effect of tower and nacelle on the flow past a wind turbine, Wind Energy 20 (2017) 1927-1939.

[16] A. Pourrajabian, P. Nazmi, M. Ahmadizadeh, D. Wood, Aero-structural design and optimization of a small wind turbine blade, Renewable Energy 87 (2016) 837-848.

[17] F. Ponta, A. Otero, L. Lago, R. A., Effects of rotor deformation in wind-turbine performance: The Dynamic Rotor Deformation Blade Element Momentum model (DRD-BEM), Renewable Energy 92 (2016) 157-170.

[18] M. Hand, D. Simms, L. Fingersh, D. Jager, J. Cotrell, S. Shreck, S. Larwood, Unsteady Aerodynamics Experiment Phase VI: Wind Tunnel Test Configurations and Available Data Campaigns, Tech. Rep., National Renewable Energy Laboratory, 2001.

[19] D. Simms, S. Schreck, M. Hand, L. Fingersh, NREL Unsteady Aeordynamics Experiment in the NASA-Ames Wind Tunnel: A Comparison of Predictions to Measurements, Tech. Rep., National Renewable Energy Laboratory, 2001.

[20] V. Esfahanian, P. Salavati, I. harsini, A. Shabazi, G. Ahmadi, Numerical analysis of flow field around NREL Phase II wind turbine by a hybrid CFD/BEM method, Journal of Wind Engineering and Industrial Aerodynamics 120 (2013) 29-36.

[21] M. Yelmule, V. EswaraRao, CFD predictions of NREL phase VI rotor experiments in NASA/AMES wind tunnel, International Journal of Renewable Energy Research 3 (2013) 261-269.

[22] Y. Chen, Y. Shiah, Experiments on the Performance of Small Horizontal Axis Wind Turbine with Passive Pitch Control by Disk Pulley, Energies 9 (2016) 353.

[23] H. Madsen, C. Bak, M. Dssing, R. Mikkelsen, S. ye, Validation and modification of the Blade Element Momentum theory based on comparisons with actuator disc simulations, Wind Energy 13 (2010) 373-389.

[24] M. Hansen, J. Srensen, S. Voutsinas, N. Srensen, H. Madsen, State of the art in wind turbine aerodynamics and aeroelasticity, Progress in Aerospace Sciences 42 (2006) 285-330.

[25] D. Somers, Design and Experimental Results for the S809 Airfoil, Tech. Rep., National Renewable Energy Laboratory, 1997.

[26] O. de Vries, Fluid Dynamic Aspects of Wind Energy Conversion, Tech. Rep., Advisory Group for Aerospace Research and Development, North Atlantic Treaty Organization, 1979.

[27] E. Hau, Wind Turbines, Springer, 2013

[28] S. Pope, Turbulent Flows, Cambridge University Press, 2009

[29] G. Batchelor, An Introduction to Fluid Dynamics, Cambridge University Press, 1967.

[30] M. Moshfegui, Y. Song, Y. Xie, Effects of near-wall grid spacing on SST-k- $\omega$ model using NREL Phase VI horizontal axis wind turbine, Journal of Wind Engineering and Industrial Aerodynamics 107-108 (2012) 94-105.

[31] F. Menter, Zonal two-equation $k-\omega$ turbulence model for aerodynamic flows, AIAA, Orlando, Florida 93 (1986) 93-2906

[32] D. Wilcox, Multiscale model for turbulent flows, Proceedings of the 24th AIAA Aerospace Science Meeting 24 (1986) 1311-1320.

[33] T. Burdett, K. W. VanTeuren, A Theoretical and Experimental Comparison of Optimizing Angle of Twist Using BET and BEMT, ASME Turbo Expo: Turbine Technical Conference and Exposition 2012 (2012) 797-809.

[34] D. Spera, Wind Turbine Technology, ASME Press, 2009.

[35] Y. He, R. Agarwal, Shape optimization of NREL S809 airfoil for wind turbine blades using a multiobjective genetic algorithm, International Journal of Aerospace Engineering 2014 (2014) 2845.

[36] R. Singh, R. Ahmed, Blade design and performance testing of a small wind turbine rotor for low wind speed applications, Renewable Energy 50 (2013) 812-819.

[37] A. Tummala, R. K. Velamati, D. K. Sinha, V. Indraja, V. Krishna, A review on small scale wind turbines, Renewable and Sustainable Energy Reviews 56 (2016) 1351-1371.

[38] Y. Li, K. Paik, T. Xing, P. Carrica, Dynamic overset CFD simulations of wind turbine aerodynamics, Renewable Energy 37 (2012) $285-298$.

[39] S. Lee, S. Park, K. Lee, S. Chung, Performance prediction of NREL (National Renewable Energy Laboratory) Phase VI blade adopting blunt trailing edge airfoil, Energy 47 (2012) 47-61.

[40] J. G. Leishman, Principles of Helicopter Aerodynamics, Cambridge University Press, 2006.

[41] G. Barenblatt, Scaling laws for fully developed turbulent shear flows. Part 1. Basic hypotheses and analysis, Journal of Fluid Mechanics 248 (1993) 513-520.

[42] N. Sørensen, J. Michelsen, S. Schreck, Navier-Stokes predictions of the NREL phase VI rotor in the NASA Ames $80 \mathrm{ft} x 120 \mathrm{ft}$ wind tunnel, Wind Energy 5 (2002) 151-169.

[43] L. Oggiano, CFD Simulations on the NTNU Wind Turbine Rotor and Comparison with Experiments, Energy Procedia 58 (2014) $111-116$.

[44] B. Plaza, R. Bardera, S. Visiedo, Comparison of BEM and CFD results for MEXICO rotor aerodynamics, Journal of Wind Engineering and Industrial Aerodynamics 145 (2015) 115-122. 
[45] E. Muljadi, K. Pierce, P. Migliore, Control strategy for variable-speed, stall-regulated wind turbines, Proceedings of the American control conference 3 (1998) 1710-1714.

[46] E. Muljadi, K. Pierce, P. Migliore, Soft-stall control for variable-speed stall-regulated wind turbines, Journal of Wind Engineering and Industrial Aerodynamics 85 (2000) 277-291.

[47] T. Macquart, A. Maheri, A stall-regulated wind turbine design to reduce fatigue, Renewable Energy 133 (2019) 964-970.

\section{Nomenclature}

$r \quad$ Distance to the hub

$\theta \quad$ Blade pitch angle

$c \quad$ Section chord

$V_{z} \quad$ Wind velocity

$\Omega \quad$ Wind turbine rotational speed

$v_{i} \quad$ Axial induced velocity

$a \quad$ Axial induced velocity factor

$\omega^{\prime} \quad$ Tangential induced velocity

$a^{\prime} \quad$ Tangential induced velocity factor

$\varphi \quad$ Relative velocity angle

$V_{\text {rel }} \quad$ Relative velocity

$F_{N} \quad$ Normal force

$P \quad$ Power

$Q \quad$ Torque

$C_{l} \quad$ Airfoil lift coefficient

$C_{d} \quad$ Airfoil drag coefficient

$\alpha \quad$ Angle of attack

$\lambda \quad$ Tip speed ratio

$F \quad$ Tip losses factor

$x \quad$ Non-dimensional distance

$C_{T} \quad$ Normal force coefficient

$C_{P} \quad$ Power coefficient

$C_{Q} \quad$ Torque coefficient

$c_{p} \quad$ Pressure coefficient

$\rho \quad$ Free stream density

$R \quad$ Blade radius

$B \quad$ Number of blades

$U_{i} \quad$ Mean velocity component on the $i^{\text {th }}$ direction

$v \quad$ Free stream kinematic viscosity

$\mu \quad$ Free stream dynamic viscosity

$\vec{f} \quad$ Inertial forces vector

$\vec{r} \quad$ Position vector

$\vec{\omega} \quad$ Moving Reference Frame rotational velocity

$\left\langle u_{i} u_{j}\right\rangle \quad$ Reynolds stresses

$v_{T} \quad$ Turbulent viscosity

$\delta_{i j} \quad$ Dirac delta

$\sigma \quad$ Local solidity

$u_{\text {tau }} \quad$ Shear velocity

$y^{+} \quad$ Non-dimensional wall distance

Re Reynolds number

$C_{p} \quad$ Pressure coefficient

$p \quad$ Absolute static pressure

$p_{\infty} \quad$ Free stream absolute static pressure 


\section{List of Figures}

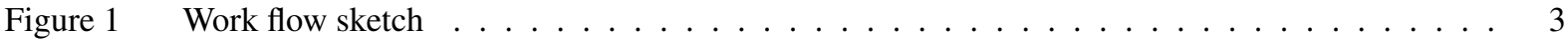

Figure 2 Velocity diagram used for the BEMT explanation at an airfoil section located at radius $r$. . 4

Figure 3 Work flow of the proposed Design of Experiments . . . . . . . . . . . . . . . . 7

Figure 4 Power (left) and force (right) curves calculated for the blade NREL Phase VI using different

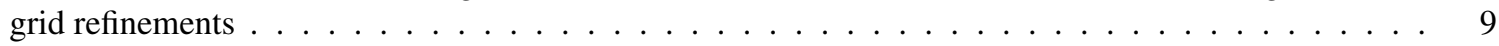

Figure 5 Wall $\mathrm{y}+$ distribution for $\lambda=3$ (left) and $\lambda=6$ (right). Calculations performed with the NREL Phase VI blade with $N \approx 8 \cdot 10^{6} \ldots \ldots \ldots \ldots \ldots \ldots \ldots \ldots$

Figure 6 Mesh sketch for a case of a three bladed wind turbine calculation . . . . . . . . . . . 10

Figure 7 Pressure coefficient. Comparison between experiments and CFD . . . . . . . . . . . . 11

Figure 8 Velocity fields arround the NREL Phase VI geometry . . . . . . . . . . . . . . . . . 12

Figure 9 Power coefficient curve. Comparison between current methodologies and experiments . . . . . 13

Figure 10 Power (left) and axial force (right) coefficient curves computed for the blade NREL Phase VI.

CFD calculations at different inlet velocities . . . . . . . . . . . . . . . . . . . . . . 13

Figure 11 Power curves for different optimization parameters . . . . . . . . . . . . . . . . . . . . 14

Figure 12 Local solidity (left) and torsion (right) distributions for two different resulting geometries from

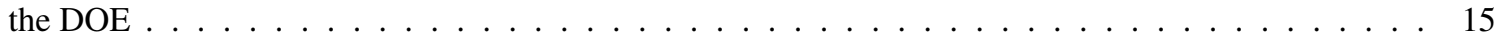

Figure 13 Comparison between the CFD and BEMT predicted power curves for the current proposed geometry (left) and BEMT prediction of the angle of attack for two different tip speed ratio (right) $\ldots 15$

Figure 14 Comparison between current design (left) and a commercial geometry (right) . . . . . . . . . 16

Figure 15 Comparison of the power curve for the different analyzed blades . . . . . . . . . . . . . 17

Figure 16 Contours of the pressure coefficient for the current design (up); the Skystream commercial blade (middle) and the Skystream commercial blade with the VG removed at the value of tip speed ratio of maximum power (left) and a low tip speed ratio (right) and stream lines of wall shear stress. $\quad .18$

Figure 17 Evolution of both non dimensional power and torque for the current design (gray), the commercial blade (red) and an alternative discarded design (green). Predictions made with RANS . . . .

Figure 18 Evolution of both dimensional power and torque for the current design (gray), the commercial blade (red) and an alternative discarded design (green) with wind velocity. Numerical application supposing wind turbines of similar radius $R=2.50 \mathrm{~m}$ working under normal conditions with $\rho_{\infty}=$ $1.225 \mathrm{~kg} \mathrm{~m}^{-3} \ldots \ldots \ldots \ldots \ldots \ldots \ldots \ldots \ldots \ldots$

Figure 19 Evolution of the power (left) and torque (right) of the discarded design as a function of maximum control rotational velocity for a given radius of $R=2.50 \mathrm{~m} \ldots \ldots \ldots$ 